\title{
Investigations of Anharmonic Low-Frequency Oscillations in Heme Proteins ${ }^{\dagger}$
}

\author{
Florin Rosca, Anand T. N. Kumar, Dan Ionascu, Xiong Ye, Andrey A. Demidov, \\ Theodore Sjodin, David Wharton, Douglas Barrick, \\ Takashi Yonetani," and Paul M. Champion*
}

Physics Department and Center for Interdisciplinary Research on Complex Systems, Northeastern University, Boston, Massachusetts 02115

Received: July 30, 2001; In Final Form: September 25, 2001

\begin{abstract}
The technique of femtosecond coherence spectroscopy is applied to a variety of photostable and photochemically active heme protein samples. With the exception of cobalt-substituted myoglobin, strong oscillations are detected near $40 \mathrm{~cm}^{-1}$ in all of the samples studied. Additional modes near 80, 120, and $160 \mathrm{~cm}^{-1}$ are observed in the photochemically active samples. The amplitude and phase behavior of the low-frequency modes are studied by tuning the pump/probe carrier wavelength across the Soret absorption spectrum. A simple harmonic model is not able to account for the observed relative intensities of these modes or the carrier wavelength dependence of their frequency and phase. As a result, we develop an anharmonic model where the oscillatory signal is damped as the result of heterogeneity in the potential surface. The underlying source of the heterogeneity in the anharmonic potential surface is found to be correlated with the inhomogeneous broadening of the Soret band. The presence of the higher harmonics in the photochemically active samples demonstrates that the anharmonic mode is strongly coupled to the ligand photodissociation reaction (i.e., upon photolysis it is displaced far from equilibrium). Moreover, the observation of the $\sim 40 \mathrm{~cm}^{-1}$ oscillations in all of the ironbased heme protein samples, including porphine and protoporphyrin IX model compounds, suggests that this mode is associated with nuclear motion of the core of the porphyrin macrocycle. Since normal mode calculations and prior kinetic models predict the frequency of the heme "doming mode" to be near $50 \mathrm{~cm}^{-1}$, we suggest that the reaction coupled oscillations at $\sim 40$ and $\sim 80 \mathrm{~cm}^{-1}$ are a direct reflection of anharmonic heme doming dynamics. Evidence for coupling between the heme doming dynamics and the $\mathrm{Fe}-\mathrm{His}$ stretching mode is also presented.
\end{abstract}

\section{Introduction}

Femtosecond coherence spectroscopy (FCS) is a pump/probe technique that utilizes the spectral bandwidth associated with ultrafast laser pulses to prepare and monitor coherent states in resonant and nonresonant samples. ${ }^{1-12}$ This technique allows the real-time observation of ultrafast processes, so that the initial phases of the detected vibrational oscillations can be measured and compared. Following the pump pulse excitation, two types of processes can occur that we refer to as "field driven" and "reaction driven". In the first case, the pump pulse excites the Raman active modes of the sample without affecting its structure. In the "reaction driven" case, the photoexcited state rapidly decays, via nonradiative channels, into a coherent "product state". In both cases, the delayed probe pulse monitors the pump-induced transient absorption changes in the sample.

In this work, we focus on the detection and characterization of time-resolved low-frequency vibrational modes in heme proteins. The heme proteins form a large class of biomolecules, which are involved in a variety of important biochemical reactions such as diatomic ligand storage and transport, enzyme

\footnotetext{
† Part of the special issue "Mitsuo Tasumi Festschrift".

* To whom correspondence should be addressed. Phone: (617) 3732918. E-mail: champ@ neu.edu.

$\doteqdot$ Department of Biophysics, Johns Hopkins University, Baltimore, MD 21218.

$\S$ Department of Biochemistry, University of Illinois at UrbanaChampaign, Urbana, IL 61801.

" Department of Biochemistry and Biophysics, University of Pennsylvania, Philadelphia, PA 19104.
}

catalysis, and electron transfer. The heme group (active site) is a planar chromophore containing iron, which is bound to the "proximal" histidine (His) in Mb and to a cysteine (Cys) in cytochrome P450. The iron atom, at the center of the heme group, can reversibly bind diatomic molecules such as NO, $\mathrm{CO}$, and $\mathrm{O}_{2}$, which can in turn be photolyzed by a femtosecond laser pump pulse. The relaxation dynamics of the nonequilibrium structure generated by the ligand photodissociation reaction has been extensively studied over a wide range of time scales using a variety of techniques. ${ }^{13-24}$ Here, we use FCS to study heme proteins such as myoglobin (in native, cobalt substituted, and mutant forms), hemoglobin, cytochrome $\mathrm{P} 450_{\mathrm{CAM}}$, cytochrome $c$, and an iron protoporphyrin IX (FePPIX) model compound.

Ligand photolysis excites low-frequency heme/protein oscillations that arise from the forces associated with electronic rearrangements in the iron d-orbitals that take place when the iron atom undergoes a spin transition upon ligand dissociation. ${ }^{6,12}$ These low-frequency modes are also potentially connected to energy transport and signal transduction between the active site and the protein backbone and can thus affect the physiological functionality of the protein. One particular vibrational degree of freedom that we have suggested ${ }^{25-27}$ is highly relevant to the ligand binding and dissociation reaction is heme "doming", which corresponds to heme motion that changes the local equilibrium position of the iron atom. The iron atom changes from an in-plane position, when the diatomic ligand is bound, to an out-of-plane $(\sim 0.4 \AA)$ position in the 
unligated state, ${ }^{28}$ which is distributed and dependent on the surrounding protein conformation. ${ }^{25-27}$ Normal mode calculations $^{29-31}$ and analysis of the nonexponential ligand rebinding ${ }^{23}$ in $\mathrm{Mb}$ predict ${ }^{25-27}$ that heme doming oscillations in the unligated state mode should be near $50 \mathrm{~cm}^{-1}$.

In this work, we present data displaying a strong vibrational mode near $40 \mathrm{~cm}^{-1}$, denoted as $v_{40}$, which is found in all of the iron-containing heme systems studied. Its frequency spans a $37-$ $50 \mathrm{~cm}^{-1}$ range, depending on the specific heme protein sample. An analysis of the structural differences between the samples displaying the $v_{40}$ oscillations leads us to associate the nuclear motion of this mode with the heme macrocycle. All of the photoreactive samples display at least one additional sampledependent vibrational mode near $80 \mathrm{~cm}^{-1}$, which we denote as $v_{80}$. The observation of $v_{40}$ in deoxy $\mathrm{Mb}$ and $v_{40}$ and $v_{80} \mathrm{MbNO}$ was reported earlier ${ }^{6,32}$ using both detuned and open band detection. Here, we study the amplitude and phase behavior of $v_{40}$ and $v_{80}$ in $\mathrm{MbNO}$ by tuning the pump/probe carrier wavelength across the Soret absorption band. A heterogeneous anharmonic potential model is used to simulate the oscillatory signals that give rise to $v_{40}$ and $v_{80}$ (and sometimes higher harmonics) in the power spectra of the photolyzed samples. These signals are assigned to motions associated with anharmonic heme doming dynamics.

\section{Materials and Methods}

We use a self-mode-locked Ti:sapphire laser (Coherent, Inc.) to generate the laser pulses used in this work. The laser generates femtosecond pulses (45-100 fs) with a $76 \mathrm{MHz}$ repetition rate and carrier wavelengths between 700 and $960 \mathrm{~nm}$. A $0.2 \mathrm{~mm}$ BBO crystal is used to double the IR light in order to obtain pump/probe pulses resonant with the Soret absorption band of the heme proteins under study $(400-450 \mathrm{~nm})$. We use anaerobic spinning cells (rotating at $\sim 1000$ and $\sim 3500 \mathrm{rpm}$ ) to refresh the sample in the illuminated volume. The average power of blue light at the sample is $30 \mathrm{~mW}$, which corresponds to about $0.4 \mathrm{~nJ} /$ pulse pair. Since a pump/probe pulse pair affects less than $1 \%$ of the sample, the possibility of buildup of the unligated species in the NO bound samples (for which the $\sim 100$ ps geminate recombination has $96-100 \%$ amplitude) is eliminated.

The pump/probe setup has been described in detail elsewhere..$^{32,33}$ To both pump and probe the studied samples, we tune the laser carrier frequency into resonance with the strong Soret absorption band in the $400-450 \mathrm{~nm}$ region. This allows us to selectively probe the dynamics of the active site (heme) and its interaction with the protein. For all experiments presented in this paper, we use the open band detection scheme in which the full spectrum of the probe beam is detected by a photodiode. This detection scheme facilitates accurate phase measurements for the oscillatory signal because the autocorrelation measurement that defines the pump/probe zero time delay also uses the open band detection.

Horse heart myoglobin, horse heart cytochrome $c$, and microperoxidase (MP-8) were purchased from Sigma Chemical. Bovine hemin (FePPIX model compound) was purchased from Aldrich Chemical Co., Inc., and octaethylporphyrin (OEP) was purchased from Porphyrin Products. The hemoglobin sample was provided Prof. J. M. Manning (Northeastern University, Biology Department). The P450 sample, ${ }^{34}$ the H93G Mb sample ${ }^{35,36}$ (proximal histidine replaced by glycine), the cobaltsubstituted $\mathrm{Mb},{ }^{37}$ and porphine-substituted $\mathrm{Mb}^{38}$ were all prepared according to standard procedures. The samples were adjusted in concentration so that the optical density at the Soret maximum was near to $1 \mathrm{OD}$ in a $0.5 \mathrm{~mm}$ sample cell.
The ferrous- and NO-bound samples are prepared in a glovebox to prevent unwanted contamination from molecular oxygen. The ferrous species was obtained by adding $2 \mu \mathrm{L}$ of 1 $\mathrm{M}$ sodium dithionite solution (ascorbic acid for cytochrome $c$ ) to $90 \mu \mathrm{L}$ of buffered sample. An additional $1 \mu \mathrm{L}$ of $1 \mathrm{M} \mathrm{NaNO}_{2}$ solution is added to prepare the NO adduct. An alternative way to prepare the $\mathrm{NO}$ adduct is through the addition of $\mathrm{NO}$ gas at atmospheric pressure. The latter method provides a 10-100fold higher concentration of $\mathrm{NO}$ in solution than the former, which affects the NO bimolecular recombination rates. The nitric oxide ligand is used in this study because it geminately rebinds to the heme iron in 10-100 ps, allowing for a complete reset of the sample prior to the arrival of the next pump/probe pulse pair.

Potassium phosphate buffer $(\mathrm{pH} 7.8,0.1 \mathrm{M})$ is used for all samples unless otherwise indicated. The P450 $\mathrm{CAM}$ was dissolved in $0.1 \mathrm{M}, \mathrm{pH} 7.4$, potassium phosphate buffer with the addition of $1 \mathrm{mM}$ d-camphor for the preparation of camphor bound P450 ${ }_{\text {CAM }}$. The Fe protoporphyrin IX (FePPIX) solution was prepared with the addition of 2-methylimidazole (2MeIm) (which coordinates to $\mathrm{Fe}$ ) and $1.5 \%$ cetyl trimethylammonium bromide (CTAB), to prevent sample aggregation. The octaethylporphyrin (OEP) was diluted in an aqueous sodium dodecyl sulfate (SDS) detergent micellar solution, ${ }^{39}$ with the addition of 2 M 2-methylimidazole (which coordinates to Fe). The microperoxidase-8 (MP8) solution was prepared with the addition of $1.5 \%$ detergent (CTAB), to prevent sample aggregation. The H93G sample contains 2 M 2-methylimidazole, which complexes to the heme in place of the proximal histidine ligand.

The concentration of protein is chosen so that the sample has an absorbance of about 0.6 OD at the pump wavelength in a $0.5 \mathrm{~mm}$ cell. Each data set is the average of $60-120$ scans of the optical delay line, generated in a $2-4 \mathrm{~h}$ experiment. To ensure sample integrity, the absorption spectrum of the sample is measured before and after each experiment and intermediate FCS files are routinely compared in order to gauge the sample stability during the course of the experiment.

Typical measured signals for resonant samples consist of damped oscillations superimposed on a monotonically decaying background. The coherence coupling signal that dominates the signal around the zero time delay is truncated before data analysis. To generate the power spectrum associated with the time-resolved oscillatory signal, we use a linear predictive singular value decomposition (LPSVD) algorithm ${ }^{40}$ that simultaneously fits both the oscillations and the monotonic decay. This algorithm extracts the oscillatory parameters such as frequency, phase, amplitude, and damping factors, as well as the exponential parameters of the (zero frequency) monotonic background. An alternative method of analysis involves preliminary fitting of the monotonic background using the maximum entropy method (MEM), which uses a distribution of exponential decay rate constants. ${ }^{41}$ The residual oscillatory signal is subsequently fit using LPSVD or a Fourier transform algorithm. The fits are considered stable when both techniques generate the same power spectrum for the oscillatory signals.

\section{Experimental Results}

To facilitate the interpretation of the experimental results, we briefly describe the active site (heme) structure and its links to the surrounding protein. The heme (iron protoporphyrin IX) is the active site of $\mathrm{Mb}$ and contains a porphyrin macrocycle with substituent groups bound to it (see Figure 1). The porphyrin macrocycle is a ringed structure containing 20 carbon atoms bound to an inner ring of four nitrogens. Off the outer macro- 


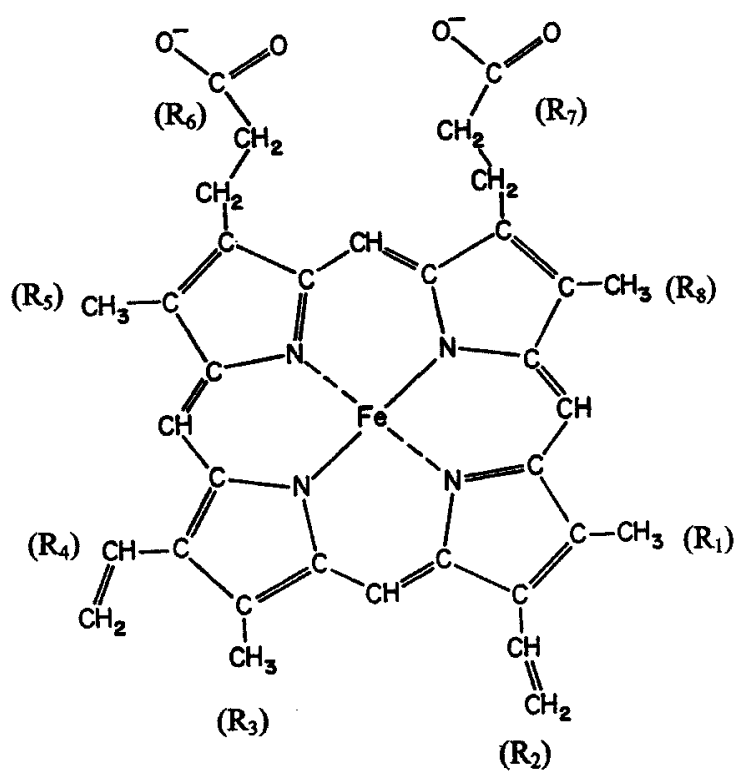

Figure 1. Structure of iron protoporphyrin IX (FePPIX). FePPIX is an asymmetric porphyrin with two vinyl groups bound at $R_{2}$ and $R_{4}$, and two propionic acid groups at $\mathrm{R}_{6}$ and $\mathrm{R}_{7}$.

cycle, there are eight sites where substituent groups can be bound $\left(\mathrm{R}_{1}-\mathrm{R}_{8}\right)$. Iron protoporphyrin IX (FePPIX) is an asymmetric porphyrin with two vinyl groups bound at $R_{2}$ and $R_{4}$ and two propionic acid groups at $\mathrm{R}_{6}$ and $\mathrm{R}_{7}$. Horse heart myoglobin, hemoglobin, and cytochrome P450 ${ }_{\mathrm{CAM}}$ all contain an FePPIX active site, which is covalently bound to the protein solely by an iron-histidine (or iron-cysteine for $\mathrm{P} 450_{\mathrm{CAM}}$ ) coordination. H93G myoglobin is a myoglobin mutant for which the axial heme ligand (histidine 93) is replaced by a glycine, such that the heme-protein covalent bond is broken. In porphine $\mathrm{Mb}$, the protoporphyrin IX is replaced by a porphine macrocycle with no substituent groups. ${ }^{38}$ In horse heart cytochrome $c$, the FePPIX structure is perturbed by the thioether bridges that bind to the heme (at $R_{2}$ and $R_{4}$ ) from two cysteine side chains (Cys14 and Cys17). The iron atom is also coordinated to a histidine (His 18) and methionine (Met 80). Octapeptide (microperoxidase-8) is produced from the digestion of horse heart cytochrome $c$ to a peptide chain of residues 14-21. The five-coordinate heme is still bound to the peptide by two thioether bridges but only histidine 18 coordinates to the iron atom.

A. Photostable Samples. Using the spectral bandwidth associated with the 50-100 fs pulses, we can excite and monitor vibrational modes below $400 \mathrm{~cm}^{-1}$. Since we try to enhance the low-frequency part of the vibrational spectrum, we typically use the longest pulses (80-100 fs) (corresponding to the narrowest bandwidth) generated by the laser. The open band detection, in contrast to the highly detuned detection used in earlier studies, ${ }^{6}$ also enhances the low-frequency modes. ${ }^{32,42}$ When using photostable samples (such as deoxy $\mathrm{Mb}$ ), the coherent oscillations are primarily induced by the forces developed during the pump pulse, which are associated with the differences between the ground and resonant excited electronic state potential energy surfaces. However, pumpinduced electronic internal conversion (nonradiative population decay) processes cannot be completely ruled out as a potential source of impulsive forces that drive low-frequency modes. Since for heme proteins the resonant $\pi-\pi^{*}$ Soret excited state is very short-lived and the population decay takes place within $10-100 \mathrm{fs},{ }^{43-47}$ we assign the long-lived $(\sim 1-2 \mathrm{ps})$ oscillatory signals to the ground electronic state. This assignment is verified
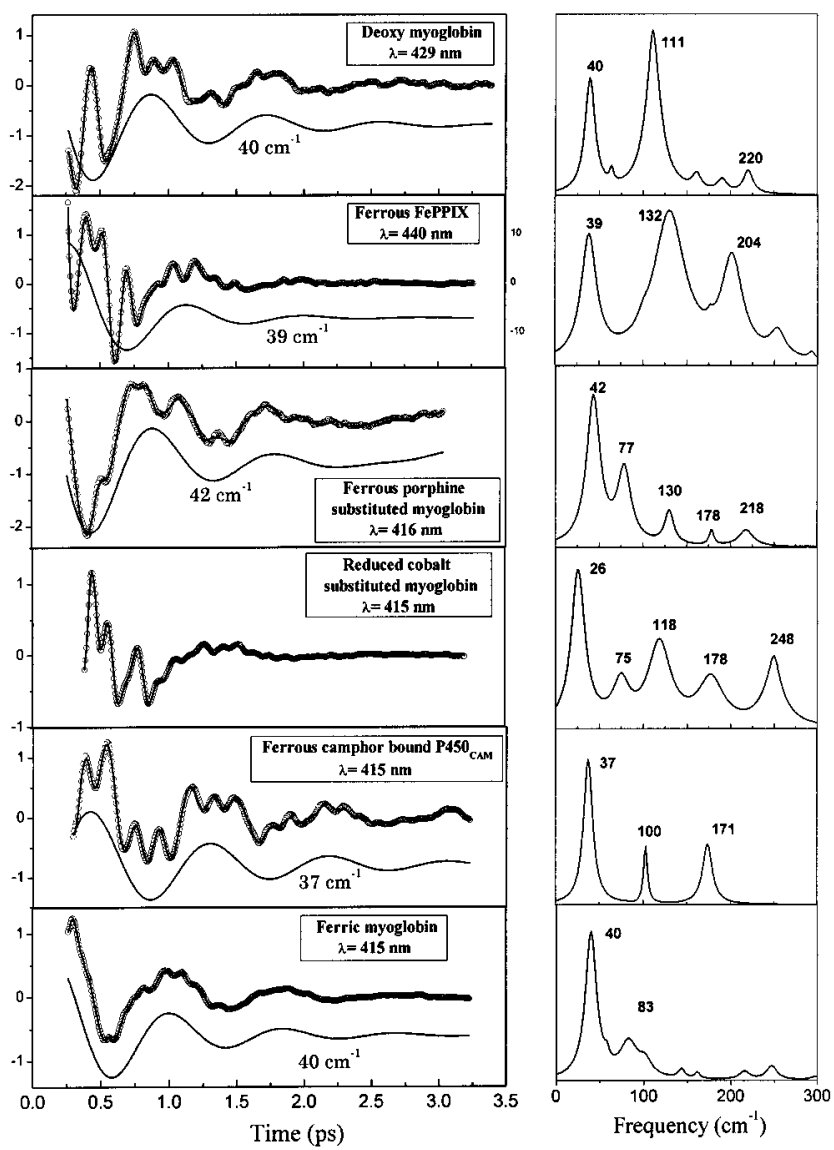

Figure 2. Sequence of experiments using the open band detection scheme on six different heme proteins. The left panels present the oscillatory part of the generated signal, and the right panels show the corresponding power spectra. The $37-40 \mathrm{~cm}^{-1}$ oscillation used in the fit is shown displaced from the data as a solid line. The sample and the pump/probe carrier wavelength are given in the right upper corner of the left panels.

by white light continuum measurements of the transient absorption spectrum in the Soret region. ${ }^{48}$

The presence of a $\sim 40 \mathrm{~cm}^{-1}$ mode can be clearly seen in the oscillatory signal of deoxy $\mathrm{Mb}$ and in the LPSVD generated power spectrum presented in the first panel of Figure 2. To characterize the heme/protein dynamics associated with this mode, we have performed experiments on a variety of other heme protein samples. Data generated in some of the other photostable samples are also displayed in Figure 2. The (statistically averaged) results obtained on all samples studied can be found in Table 1 . The observation of a mode at $39 \mathrm{~cm}^{-1}$ in ferrous FePPIX +2 MeIm (second panel of Figure 2), which contains no surrounding protein, suggests that $v_{40}$ involves heme-specific motions. The faster decay (i.e., damping) of the $39 \mathrm{~cm}^{-1}$ mode in FePPIX compared to the $40 \mathrm{~cm}^{-1}$ mode of deoxy $\mathrm{Mb}$ also suggests that a higher degree of heme structural inhomogeneity or pure dephasing is present in the absence of protein material (see following discussion).

The presence of a $42 \mathrm{~cm}^{-1}$ oscillation in porphine substituted $\mathrm{Mb}$ (third panel of Figure 2) suggests that the source of the $v_{40}$ oscillation is associated with the core of the porphyrin macrocycle and does not depend strongly on the peripheral substituents. Another possible source of $v_{40}$, the torsional motion of the histidine 93, appears inconsistent with the presence of modes at 43 and $39 \mathrm{~cm}^{-1}$ in deoxy H93G Mb and FePPIX (see Table 1). A 2-methylimidazole coordinates to the iron atom in both cases, but its torsional motion is expected to be significantly 
TABLE 1: Summary of $v_{40}$ and $v_{80}$ in Various Heme Systems $^{a}$

\begin{tabular}{|c|c|c|c|c|}
\hline samples & $\begin{array}{c}v_{40} \\
\left(\mathrm{~cm}^{-1}\right)\end{array}$ & $\begin{array}{l}\gamma_{40}^{-1} \\
(\mathrm{ps})\end{array}$ & $\begin{array}{c}v_{80} \\
\left(\mathrm{~cm}^{-1}\right)\end{array}$ & $\begin{array}{l}\gamma_{80}^{-1} \\
(\mathrm{ps})\end{array}$ \\
\hline \multicolumn{5}{|l|}{ myoglobin } \\
\hline met $\mathrm{Mb}$ & 41 & 0.67 & $82(w)$ & 0.4 \\
\hline deoxy $\mathrm{Mb}$ & 41 & 0.73 & & \\
\hline $\mathrm{MbNO}$ & 41 & 0.57 & $81(\mathrm{~s})$ & 0.49 \\
\hline deoxy $\mathrm{Mb}$ in $\mathrm{D}_{2} \mathrm{O}$ & 41 & 0.7 & & \\
\hline $\mathrm{MbNO}$ in $\mathrm{D}_{2} \mathrm{O}$ & 41 & 0.54 & $81(\mathrm{~s})$ & 0.45 \\
\hline deoxy $\mathrm{Mb}$ in $75 \%$ glycerol & 41 & 0.71 & & \\
\hline MbNO in $75 \%$ glycerol & 41 & 0.65 & $81(\mathrm{~s})$ & 0.43 \\
\hline deoxy H93G Mb & 43 & 0.7 & & \\
\hline $\mathrm{H} 93 \mathrm{G} \mathrm{MbNO}$ & 43 & 0.8 & $87(\mathrm{~s})$ & 0.33 \\
\hline reduced cobalt $\mathrm{Mb}$ & & & & 0.47 \\
\hline ferrous porphine $\mathrm{Mb}$ & 42 & 0.45 & $76(w)$ & 0.56 \\
\hline \multicolumn{5}{|l|}{ hemoglobin } \\
\hline deoxy $\mathrm{Hb}$ & 46 & 0.86 & & \\
\hline $\mathrm{HbNO}$ & 46 & 0.55 & $91(\mathrm{~s})$ & 0.35 \\
\hline \multicolumn{5}{|l|}{ model compounds } \\
\hline ferrous FePPIX + 2MeIm & 39 & 0.5 & & \\
\hline ferric FePPIX + 2MeIm & 37 & 0.5 & & \\
\hline $\mathrm{CO}$ bound FePPIX $+2 \mathrm{MeIm}$ & 44 & 0.53 & $91(\mathrm{~m})$ & 0.2 \\
\hline ferrous OEP + 2MeIm & 46 & 0.6 & & \\
\hline \multicolumn{5}{|l|}{ cytochromes } \\
\hline ferrous cytochrome $c$ & 44 & 0.53 & $89(\mathrm{~m})$ & 0.5 \\
\hline ferric cytochrome $c$ & 46 & 0.6 & $95(\mathrm{~m})$ & 0.46 \\
\hline ferrous octapeptide & 50 & 0.6 & & \\
\hline ferric camphor bound $\mathrm{P} 450_{\mathrm{CAM}}$ & 40 & 0.84 & & \\
\hline ferrous camphor bound $\mathrm{P} 450_{\mathrm{CAM}}$ & 37 & 0.92 & & \\
\hline
\end{tabular}

${ }^{a}$ The estimated uncertainty for the frequencies are $\pm 2 \mathrm{~cm}^{-1}$. The associated lifetime uncertainty is about $25 \%$. The met $\mathrm{Mb}$, deoxy $\mathrm{Mb}$, and $\mathrm{MbNO}$ samples were run extensively, such that the results displayed for these samples are more accurate $\left( \pm 1 \mathrm{~cm}^{-1}\right)$ due to better statistical averaging. The intensity of $v_{80}$ is compared with the intensity of $v_{40}$, where $\mathrm{s}=$ strong, $\mathrm{m}=$ medium, and $\mathrm{w}=$ weak. The damping constant $\left[\gamma^{-1}(\mathrm{ps})\right]$ is converted to the full width at half-maximum $\left[\gamma_{\mathrm{fwhm}}\left(\mathrm{cm}^{-1}\right)\right]$ using the expression: $\gamma_{\mathrm{fwhm}}=\gamma / \pi c$.

different from that of histidine 93 . The presence of a $37 \mathrm{~cm}^{-1}$ mode in ferrous $\mathrm{P} 450_{\mathrm{CAM}}$ (fifth panel of Figure 2), in which the histidine ligand is replaced by a cysteine, definitely rules out histidine torsional motion as the source of $v_{40}$.

The only sample in which $v_{40}$ is not obviously present (the closest detected modes are at 26 and $75 \mathrm{~cm}^{-1}$ ) is the cobalt substituted $\mathrm{Mb}$ (fourth panel of Figure 2) in which the ferrous iron atom $\left(\mathrm{d}^{6}\right)$ is replaced by cobalt $\left(\mathrm{d}^{7}\right)$. This observation suggests that $v_{40}$ is somehow related to the electronic structure of the central metal. However, a comparison between the results obtained for the ferric (see the last panel of Figure 2) and ferrous states of Mb, FePPIX, cytochrome $c$, and cytochrome P450 shows that oxidation of the iron atom to $\mathrm{d}^{5}$ does not significantly affect the frequency of $v_{40}$ (see Table 1 ).

Additional studies on samples of deoxy $\mathrm{Mb}$ and $\mathrm{MbNO}$ prepared in $\mathrm{D}_{2} \mathrm{O}$ buffer and in $75 \%$ glycerol buffer display the same low-frequency mode structure as the samples prepared in normal aqueous buffer (see Table 1). Thus, we conclude that changes made to the hydration shell of the heme/protein do not have noticeable effects on the observed vibrational characteristics of the sample. The influence of the surrounding protein on the frequency of $v_{40}$ (ranging from $37 \mathrm{~cm}^{-1}$ in ferric FePPIX, to $50 \mathrm{~cm}^{-1}$ in ferrous microperoxidase) can be found in Table 1.

As mentioned earlier, the open band detection scheme facilitates accurate phase measurements for the FCS oscillatory signal. In addition, the inherent error of the zero time delay measurement has a smaller impact on the phases of the lower frequency modes compared to the higher frequency modes. On the other hand, the phases of the low-frequency modes are more

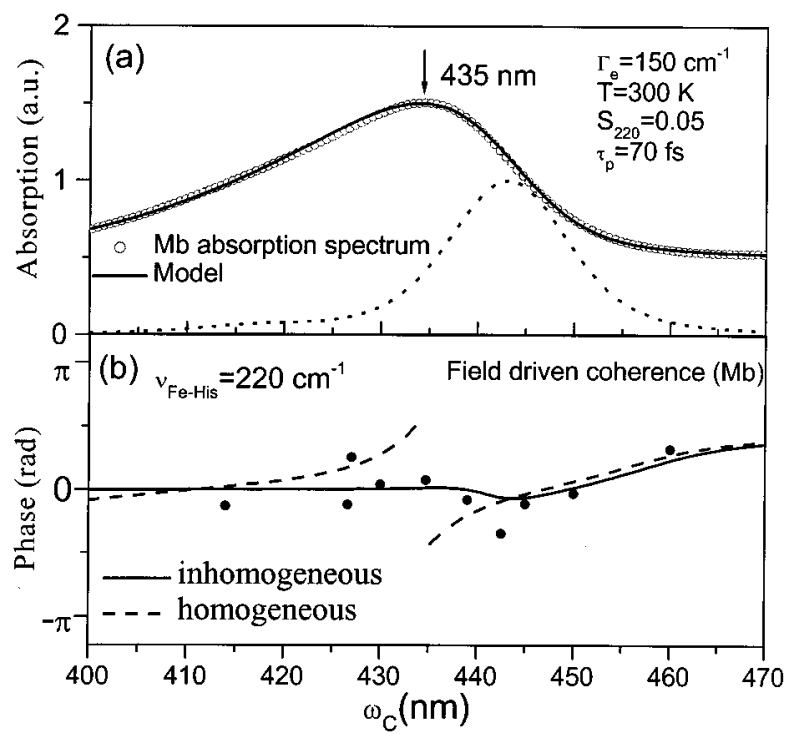

Figure 3. (a) Equilibrium absorption spectrum of deoxy Mb at $T=$ $300 \mathrm{~K}$ (open circle) shown with the theoretical fit (solid line) based on a model for the asymmetric deoxy $\mathrm{Mb}$ line shape. The underlying homogeneous line shape (before the convolution with the inhomogeneous distribution) is also shown (dotted line). (b) The measured phase profile of the $220 \mathrm{~cm}^{-1}$ mode in deoxy $\mathrm{Mb}$ is presented (circles) along with two theoretical fits, based on a homogeneous (dashed line) and an inhomogeneous (solid line) model.

likely to be affected by the uncertainties associated with the smaller number of recurrences detected for low-frequency oscillations. We consider the phase measurements presented below for $v_{40}$ and $v_{80}$ to be significant within a margin of error, estimated from the phase variation $\left( \pm 30^{\circ}\right)$ observed for similar experimental conditions. Apart from experimental considerations that favor open band over dispersed measurements for accurate phase determination, measurements of the open band oscillatory amplitude and phase over a wide range of carrier frequencies (amplitude and phase profiles) can also help assign the origin of vibrational coherences in pump/probe spectroscopy. ${ }^{49}$ For example, the degenerate pump/probe signal arising from a ground-state coherence has a phase profile that varies over a range of $2 \pi$ as the carrier frequency is tuned across the absorption spectrum, with a sharp discontinuity between $\pm \pi / 2$ at the maximum of the resonant absorption band. ${ }^{49}$ In contrast, the excited-state phase profile, is either zero or $\pi$ with a discrete phase jump at the resonant absorption band maximum.

With the above considerations in mind, we have measured the open band oscillatory amplitude and phase profiles of various modes in deoxy $\mathrm{Mb}$ and $\mathrm{MbNO}$ across the Soret absorption band. We first present, in Figure 3, the phase profile of the 220 $\mathrm{cm}^{-1}$ mode in deoxy $\mathrm{Mb}$, over the wavelength range of 400 to $460 \mathrm{~nm}$. The $220 \mathrm{~cm}^{-1}$ mode has been very well characterized by previous resonance Raman studies and is assigned to $\mathrm{Fe}-$ His stretching motion. ${ }^{50}$ In the top panel of Figure 3, we plot the measured absorption spectrum of $\mathrm{Mb}$ along with a theoretical fit based on a previously proposed model for the underlying inhomogeneous distribution of electronic transition energies. ${ }^{26,51}$ All resonance Raman active modes are included in the model, with coupling strengths determined from their absolute resonance Raman cross-sections. ${ }^{52,53}$ The degenerate pump/probe calculations are carried out using the effective linear response approach ${ }^{49}$ with a linearly displaced harmonic oscillator model for the vibrational modes. In Figure $3 b$, we plot the (open band) phase profile of the $220 \mathrm{~cm}^{-1}$ mode. The theoretical prediction for the phase of the $220 \mathrm{~cm}^{-1}$ mode in the ground state is shown for both the inhomogeneous line shape $\operatorname{model}^{26,51}$ as well as 


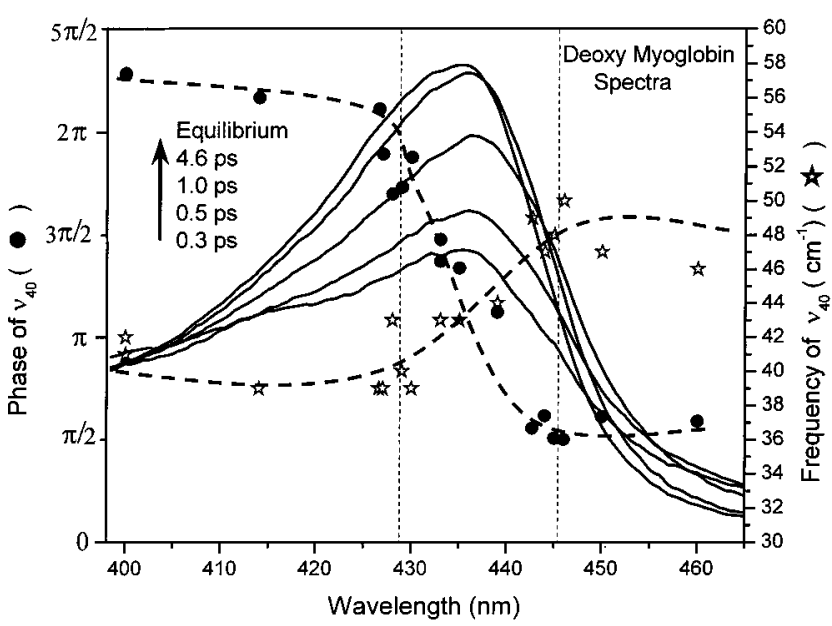

Figure 4. Phase (filled circles) and frequency (stars) profiles for $v_{40}$ in deoxy $\mathrm{Mb}$ are plotted as function of the pump/probe carrier wavelength. The vertical dashed lines delineate the region of phase and frequency drift. The pump-induced transient absorption line shape of deoxy $\mathrm{Mb}$ is also depicted for four time delays $(0.3,0.5,1$, and 4.6 $\mathrm{ps}$ ), along with the equilibrium absorption line shape.

for a homogeneous model. It is clear that the theoretical prediction using the inhomogeneous line shape is in good agreement with the experimental data. Note that the phase approaches the off-resonant limit of $\pi / 2$ much more rapidly on the red side than on the blue side of the absorption maximum. This reflects the strong asymmetry in the absorption spectrum. The sharp phase jump at the absorption maximum $(435 \mathrm{~nm})$ between $-\pi / 2$ to $+\pi / 2$ predicted by the homogeneous model is not observed in the experimental data. Also, contrary to the prediction of the homogeneous model, the experiment does not show a clear dip the amplitude of the $220 \mathrm{~cm}^{-1}$ mode near the absorption maximum. These observations are consistent with the assignment of the $220 \mathrm{~cm}^{-1}$ mode to a ground state coherence and strongly support the presence of significant inhomogeneous broadening in the Soret band of deoxy $\mathrm{Mb}$. If the $220 \mathrm{~cm}^{-1}$ coherence originated from the excited state rather than the ground state, an amplitude dip and phase flip should be observed but slightly red-shifted from the emission maximum. ${ }^{49}$ In fact, such phase behavior has been reported ${ }^{32}$ for the reaction driven $220 \mathrm{~cm}^{-1}$ coherence of $\mathrm{MbNO}$ (which is effectively an excited-state coherence).

We now turn to the behavior of $v_{40}$ in deoxy Mb. In contrast to the high-frequency oscillations at $220 \mathrm{~cm}^{-1}$, the frequency of $v_{40}$ seems to vary over the range $40-48 \mathrm{~cm}^{-1}$ as the pump/ probe carrier wavelength is tuned to the red of the Soret absorption band. This is depicted in Figure 4, where we plot the measured frequencies along with the open band phase profiles. In addition to the systematic frequency drift (star symbols), we see that the phase profile (solid dots) also exhibits a strikingly different behavior from the predictions of the linearly displaced harmonic model shown in Figure 3. Rather than remaining a constant, the phase continually drifts over a range of about $2 \pi$ in the $430-444 \mathrm{~nm}$ region.

B. Photochemically Active Samples. So far, we have been mainly concerned with nonreactive compounds, where the vibrational coherences are driven by the electric field interventions of the ultrafast pump laser pulse. Another important characteristic of time-resolved FCS is its ability to monitor chemical reactions and the subsequent evolution of the nonequilibrium product state. In this section, we present FCS experiments on a variety of photochemically active samples. The experimental data for some of these samples is plotted along with the LPSVD fits and power spectra in Figures 5-7. The most distinguishing feature of the results in Figures 5-7, compared to the nonreactive samples, is the presence of a second mode at $\sim 80 \mathrm{~cm}^{-1}$ (and sometimes higher harmonics at $\sim 120 \mathrm{~cm}^{-1}$ and $\sim 160 \mathrm{~cm}^{-1}$ ), in addition to the low-frequency mode near $40 \mathrm{~cm}^{-1}$. The mode near $80 \mathrm{~cm}^{-1}$ is significantly enhanced relative to $v_{40}$ when detuned detection conditions are employed, ${ }^{32,42,49}$ and as a result, $v_{80}$ was emphasized in earlier studies ${ }^{6}$ conducted under such conditions.

The frequencies of $v_{40}$ and $v_{80}$ for various photoreactive heme samples are presented in Table 1 . Both $v_{40}$ and $v_{80}$ show slight sample dependent variations in frequency, but the higher frequency mode remains approximately twice the frequency of the low-frequency mode. The frequency pairs observed are $41-81 \mathrm{~cm}^{-1}$ in $\mathrm{MbNO}, 46-91 \mathrm{~cm}^{-1}$ in $\mathrm{HbNO}, 44-89 \mathrm{~cm}^{-1}$ in ferrous cytochrome $c, 43-87 \mathrm{~cm}^{-1}$ in NO-bound $\mathrm{H} 93 \mathrm{G} \mathrm{Mb}$, and $44-91 \mathrm{~cm}^{-1}$ in CO-bound FePPIX. The fact that the higher frequency oscillations near $80 \mathrm{~cm}^{-1}$ are approximately twice the frequency of the oscillations near $40 \mathrm{~cm}^{-1}$ is suggestive of a harmonic sequence. In the case of $\mathrm{MbNO}$, it appears that even higher harmonics are present at 122 and $165 \mathrm{~cm}^{-1}$ (see top panel of Figure 7 and previous reports). ${ }^{12,32}$

Furthermore, the low-frequency oscillations seen in the MbNO sample near $40 \mathrm{~cm}^{-1}$ exhibit a continuous drift from 40 to $46 \mathrm{~cm}^{-1}$, as the laser carrier wavelength is tuned toward the red $(450-470 \mathrm{~nm}$ ) of the product state (deoxy $\mathrm{Mb}$ ) Soret absorption band. This frequency drift is shown in the upper panel of Figure 6 and follows the same general pattern observed for the field driven coherence in deoxy Mb (Figure 4). Note that, as for different heme samples, the frequency of the oscillations near $80 \mathrm{~cm}^{-1}$ remains approximately twice the frequency of the $40 \mathrm{~cm}^{-1}$ mode. These observations strongly suggest that $v_{80}$ forms part of a harmonic progression with $v_{40}$ as the fundamental frequency.

Another example of photochemical activity is ferrous cytochrome $c$, which was recently shown ${ }^{54}$ to undergo a rapid ligand (methionine) photodissociation reaction followed by $\sim 6 \mathrm{ps}$ geminate rebinding. The oscillatory part of the FCS signal of ferrous cytochrome $c$ is presented at four different carrier wavelengths in the first four panels of Figure 5, along with the corresponding LPSVD power spectra. The main features observed in the signal are a damped $\sim 44 \mathrm{~cm}^{-1}$ oscillation $\left(v_{40}\right)$ and a $\sim 89 \mathrm{~cm}^{-1}$ oscillation $\left(\nu_{80}\right)$ of smaller amplitude. Both of these modes exhibit a $\pi$ phase flip and a dip in amplitude around $423 \mathrm{~nm}$, which is close to the product state transient absorption maximum near $425 \mathrm{~nm}$ (known from white light continuum experiments). ${ }^{54}$ The amplitude of $v_{80}$ seems to decrease faster than $v_{40}$ as the laser pulse carrier wavelength approaches 423 $\mathrm{nm}$. The data generated at $423 \mathrm{~nm}$ are not shown because of the lack of an oscillatory signal. The $\sim 44$ and $\sim 89 \mathrm{~cm}^{-1}$ oscillations used in the LPSVD fit to the data are depicted as solid and dashed lines, respectively (shifted from the data for clarity). The two vertical lines going through all panels are helpful in comparing the relative phases of the oscillatory components. An important observation is the coincidence of the $\sim 89 \mathrm{~cm}^{-1}$ oscillation maxima or minima with the maxima and minima of the $\sim 44 \mathrm{~cm}^{-1}$ oscillation and the fixed relative phase at all carrier wavelengths.

Turning back to MbNO, we present in Figure 6 the phase profiles of $v_{40}$ and $v_{80}$, as the laser carrier frequency is varied across the Soret absorption region from 400 to $480 \mathrm{~nm}$. The phase behavior of the two modes is very similar and their relative phase tracks together across the studied spectral region. The amplitude of $v_{80}$ is very small in the $420-435 \mathrm{~nm}$ region, 


\section{Ferrous cytochrome c}
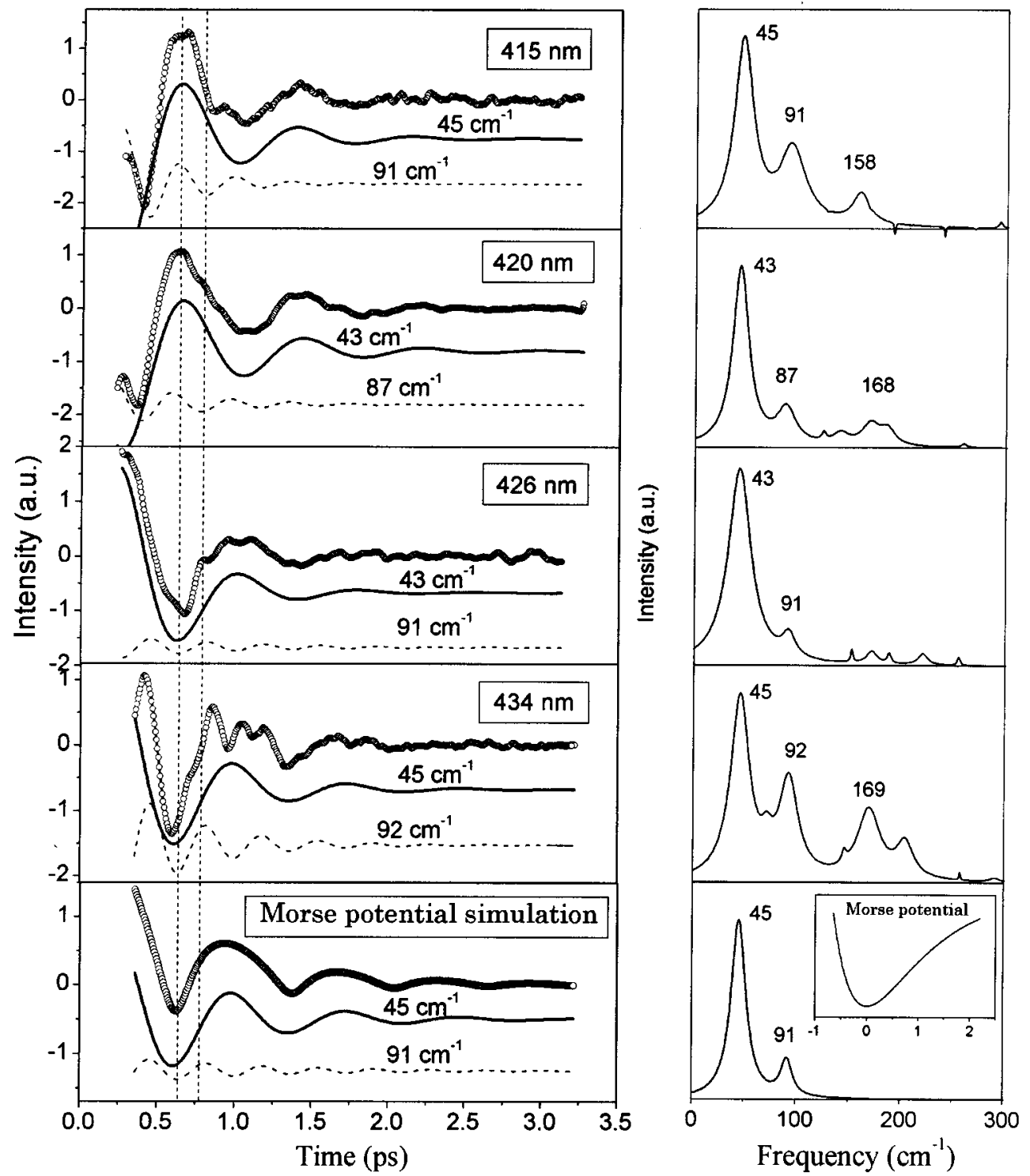

Figure 5. Sequence of experiments using the open band detection scheme on ferrous cytochrome $c$ for four pump/probe carrier wavelengths. The left panels present the oscillatory part of the signal, and the right panels show the corresponding power spectra. The $\sim 44 \mathrm{~cm}^{-1} \mathrm{oscillation}$ (solid line) and the $\sim 89 \mathrm{~cm}^{-1}$ oscillation (dashed line) used in the fit are shown displaced from the data. One can observe a $\pi$ phase flip for both modes in the $420-426 \mathrm{~nm}$ spectral region. The left lower panel displays the simulated signal generated by the dynamics of a particle in a Morse potential $\left[U_{\mathrm{g}}(q)=\mathrm{e}^{-2 q}-2 \mathrm{e}^{-q}\right]$, along with the 45 and $91 \mathrm{~cm}^{-1}$ oscillations used for its fit. The lower right panel presents the associated power spectrum and a plot of the Morse potential (in the inset).

such that the corresponding phases are difficult to extract and hence not plotted for this wavelength region. We also plot the ratio of the amplitudes of the two modes, $I_{80} / I_{40}$, in the lower panel of Figure 6. The dip of the amplitude ratio near $427 \mathrm{~nm}$ is evident.

The oscillatory parts of the open band FCS signals in three different photochemically active samples (MbNO, NO-bound H93G Mb, and CO-bound FePPIX + 2MeIm) are displayed in Figure 7, along with the corresponding power spectra. The CObound FePPIX model complex was studied using a lower repetition rate amplified laser system ${ }^{33}$ so that sample reset would be assured between the arrival of the pump/probe pulse pairs. The $v_{40}$ and $v_{80}$ oscillations used in fitting the data are depicted as solid and dashed lines (shifted for clarity). The FeHis mode is found near $220 \mathrm{~cm}^{-1}$ in the power spectrum when the protein material is present and is downshifted to $211 \mathrm{~cm}^{-1}$ for the FePPIX model complex. ${ }^{55}$ The low-frequency modes present in the power spectra of $\mathrm{MbNO}$ (upper right panel in
Figure 7) seem to form a four-term harmonic sequence $(41,82$, $\left.123,164 \mathrm{~cm}^{-1}\right)$. This harmonic sequence is present in numerous data sets generated using MbNO. ${ }^{12,32}$

\section{Discussion}

The technique of FCS is very useful for monitoring the nuclear motions associated with biomolecular reaction dynamics, especially in the low-frequency region of the vibrational spectrum. We have documented the presence of a mode near $40 \mathrm{~cm}^{-1}\left(v_{40}\right)$ in all of the photostable heme proteins studied except cobalt-substituted $\mathrm{Mb}$. In addition, we have found that the photochemically active heme samples display a harmonic progression involving this mode. The frequency, phase, and amplitude dependence of $v_{40}$ and $v_{80}$ were studied for deoxy $\mathrm{Mb}, \mathrm{MbNO}$, and ferrous cytochrome $c$ as a function of the pump/probe carrier wavelength.

A. Phase and Frequency of $\boldsymbol{v}_{\mathbf{4 0}}$. For deoxy Mb, we observe a discrepancy between the phase behavior of $v_{40}$ and the 


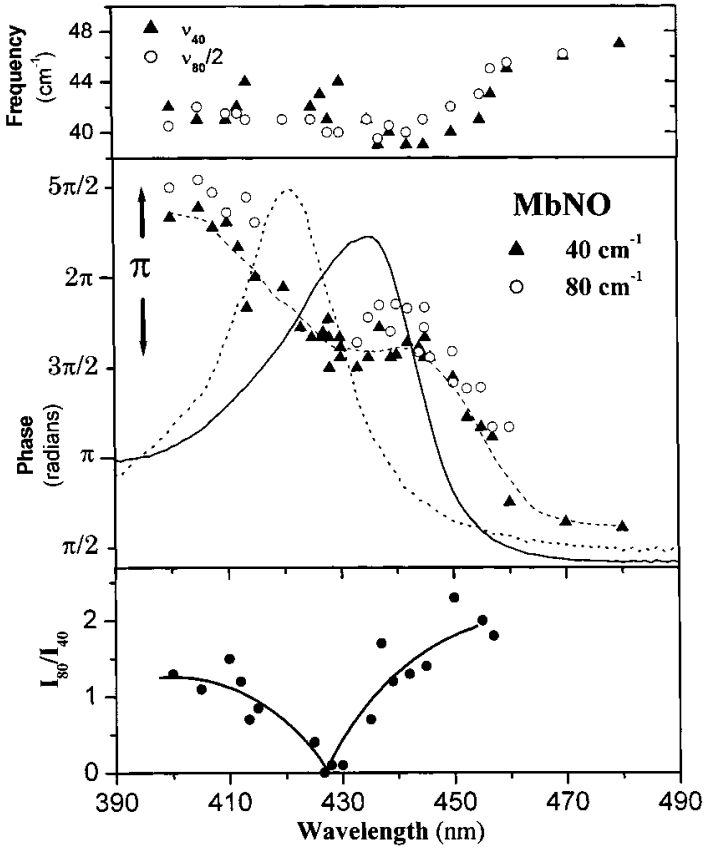

Figure 6. Upper panel displays the frequency of $v_{40}$ and $v_{80}$ in $\mathrm{MbNO}$, as the pump/probe carrier wavelength is scaned over the $400-480 \mathrm{~nm}$ spectral region. Half the frequency of $v_{80}$ is plotted to facilitate its comparison with the frequency behavior of $v_{40}$. The middle panel displays the phase behavior of $v_{40}$ (triangles) and $v_{80}$ (circles) modes in $\mathrm{MbNO}$, along with the equilibrium absorption line shapes of $\mathrm{MbNO}$ (dashed line) and deoxy $\mathrm{Mb}$ (solid line). In the lower panel, the intensity ratio of $v_{80}$ and $v_{40}$ is displayed, showing a dip in the amplitude of $v_{80}$ near $427 \mathrm{~nm}$.
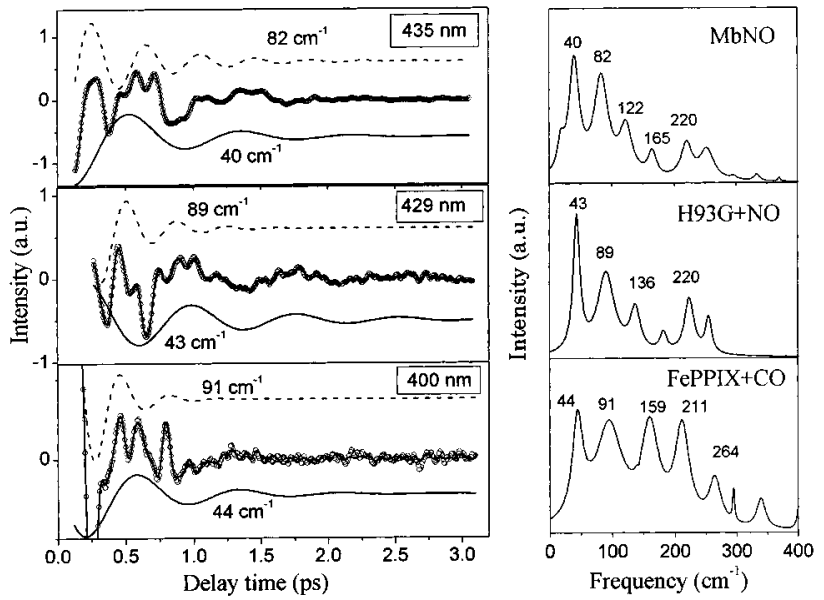

Figure 7. Sequence of experiments using the open band detection scheme on three photochemically active samples (MbNO, NO-bound H93G Mb, and CO bound FePPIX + 2MeIm). The left panels display the oscillatory part of the generated signal, and the right panels show the corresponding power spectra. The $\sim 40 \mathrm{~cm}^{-1}$ oscillation (solid line) and the $\sim 80 \mathrm{~cm}^{-1}$ oscillation (dashed line) used in the fit are shown displaced from the data. The relative damping of $v_{40}$ and $v_{80}$ can be compared by using the line widths of the power spectrum.

theoretical prediction ${ }^{49}$ based on a simple linear harmonic model (cf. Figure $3 b$ ). In contrast to $v_{40}$, the simple linear harmonic model (with inhomogeneous broadening) provides an excellent description of the phase behavior of the $\mathrm{Fe}-\mathrm{His}$ mode at 220 $\mathrm{cm}^{-1}$. One possible source for this discrepancy might have to do with the dynamic nature of the transient absorption line shape of photoexcited deoxy Mb. In Figure 4, we include the transient spectra measured using a white light continuum probe ${ }^{48}$ at four different time delays $(0.3,0.5,1.0$, and $4.6 \mathrm{ps})$, along with the equilibrium absorption spectrum of $\mathrm{Mb}$. The transient deoxy
$\mathrm{Mb}$ absorption band is broadened and initially located near 439 $\mathrm{nm}$, which is toward the red side of the equilibrium absorption spectrum. The dynamics of the transient line shape consists of two major components, namely the narrowing of the spectrum and a shift to the blue as the system relaxes to equilibrium. The time scales for the transient line-shape dynamics (narrowing and blue shifting) are $0.4-4 \mathrm{ps}$ and are attributed to the cooling and relaxation ${ }^{48}$ of the hot deoxy Mb state created by the pump pulse. Since the picosecond line-shape dynamics take place on the same time scale as the low-frequency oscillations near 40 $\mathrm{cm}^{-1}\left(T_{40}=0.8 \mathrm{ps}\right)$, the shifting absorption maximum $(439 \rightarrow$ $435 \mathrm{~nm}$ ) could conceivably affect the phase behavior of the detected oscillatory signals. Such anomalies might extend over a relatively broad range of carrier wavelengths, from $\sim 430$ to $\sim 445 \mathrm{~nm}$, due to the bandwidth ( $\sim 5 \mathrm{~nm})$ of the optical pulses and the inhomogeneity associated with deoxy $\mathrm{Mb}$ line shape. As seen in Figure 4, most of the anomalous phase drift for $v_{40}$ is localized within this spectral region, delineated by vertical dashed lines.

The drift of the frequency of $v_{40}$ as the carrier wavelength is tuned across the Soret absorption band (Figure 4) is probably linked to the inhomogeneities and protein-induced structural disorder of the heme system in deoxy $\mathrm{Mb}$. As discussed earlier, the deoxy $\mathrm{Mb}$ absorption spectrum shows strong evidence of inhomogeneous broadening $26,27,51$ and the highly asymmetric inhomogeneous line shape of $\mathrm{Mb}$ has been ascribed to disorder in the equilibrium position of the central iron atom. ${ }^{26,27,51}$ This is of relevance to the low-frequency oscillations localized at the heme, which may involve the doming motion of the iron atom. Apart from a simple distribution in the equilibrium position of the heme iron, ${ }^{27}$ the experimental data presented in Figure 4 suggest that the vibrational frequency of $v_{40}$ is also distributed. Thus, while the effect of inhomogeneities on the amplitude and phase excitation profiles of the $220 \mathrm{~cm}^{-1}$ mode appears only indirectly through the distribution in the electronic origins (Figure $3 \mathrm{~b}$ ), the $v_{40}$ frequency is evidently directly correlated with the electronic transition energy (i.e., with the disorder in the iron equilibrium position). This situation arises quite naturally if the protein conformation modulates both the equilibrium position and the curvature of the potential energy surface that governs the heme doming motion. When the carrier wavelength of the pump/probe pulse is tuned across the absorption spectrum, different conformational species are optically selected that have Soret band optical transitions (associated with the iron displacement) ${ }^{27}$ that are correlated with different doming frequencies $\left(v_{40}\right)$.

Another major topic addressed in this paper concerns the assignment of $v_{40}$ and $v_{80}$ and the question of whether these modes are part of a harmonic progression or are independent fundamentals. In early work, ${ }^{6}$ which utilized detuned detection, we assigned $v_{80}$ as the doming mode. This assignment was based upon the fact that although $v_{40}$ appeared in the power spectrum, ${ }^{6}$ it was essentially undetectable in the raw data. However, recent advances in detection technique ${ }^{32,56}$ have made the existence of $v_{40}$ unequivocal and have revealed modes at even lower frequencies. ${ }^{57}$ An important observation, which strongly indicates that $v_{80}$ is a harmonic of $v_{40}$, is the fact that $v_{80} \approx 2 v_{40}$ under a variety of conditions (e.g., as carrier wavelength is varied, or as the sample is changed). These results suggest that these two modes are not independent vibrational fundamentals and force us to look for theoretical models that are consistent with those observations.

B. Harmonic Model. In considering the possibility that the low-frequency progressions in Figures 5-7 constitute an over- 


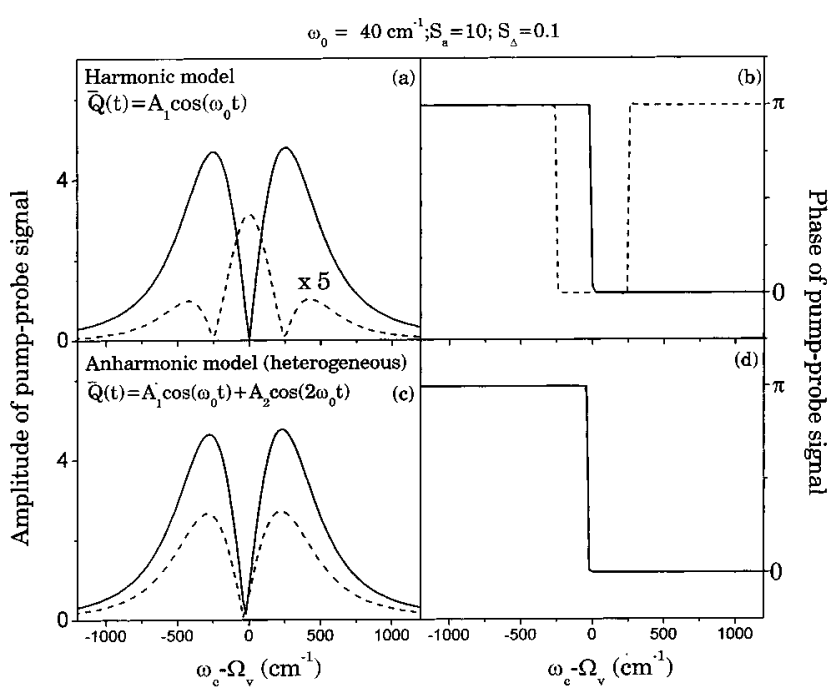

Figure 8. Comparison of the open band amplitude and phase profiles for a harmonic and anharmonic oscillator model. Panels a and b show the open band amplitude and phase profiles for the fundamental (solid) and overtone (dashed) signals for a $40 \mathrm{~cm}^{-1}$ mode coupled to a two electronic level system. Panels $\mathrm{c}$ and d show the corresponding profiles for an anharmonic model that approximates the mean position of the oscillator $Q(t)$ to be adequately described by the first two harmonics of the Fourier series, with amplitudes $A_{1}$ (solid) and $A_{2}$ (dashed). For both the simulations, the nonstationary state was assumed to be a thermal state displaced from equilibrium.

tone or harmonic sequence, one can eliminate a simple linearly displaced harmonic oscillator model. Calculations ${ }^{49}$ based on the linearly displaced harmonic model, where the main source of overtone intensity comes from the "double pass" of the wave packet across the probe detection region, predict that the intensities of the overtones are generally much smaller than the fundamental signals, as seen in the upper left panel of Figure 8 . The double pass contribution can be understood through the time-dependent first moment of the vibrational coordinate that modulates the centroid of the equilibrium absorption spectrum. For example, consider a single mode coupled to a two electronic level system, with frequency $\omega_{0}$ and electron-nuclear coupling force $f$. The pump interaction drives the nuclei out of equilibrium. If we let the time-dependent mean nuclear position after the pump interaction be $\langle Q(t)\rangle$, the correlation function that describes the response of the pump-induced nonstationary system can be shown to be of the form ${ }^{49}$

$$
C_{\text {neq }}\left(t, t^{\prime}\right)=K\left(t-t^{\prime}\right) \exp \left[\left(\frac{i f}{\hbar}\right) \int_{t^{\prime}}^{t} \mathrm{~d} s\langle Q(s)\rangle\right]
$$

where $K\left(t-t^{\prime}\right)$ is a correlation function (also called the optical absorption correlator) that describes the equilibrium line shape of the system. For a harmonic potential, the mean position $\langle Q(t)\rangle$ oscillates only at the fundamental frequency. In this case, the centroid of the nonequilibrium line shape induced by the pump pulse oscillates about the equilibrium absorption maximum at the fundamental oscillator frequency. It is then clear that the amplitude of the fundamental signal is proportional to the derivative of the absorption line shape, so that the oscillatory amplitude is largest near the shoulders of the absorption spectrum but vanishes at band center. On the other hand, the overtone amplitude profile is related to the second derivative of the absorption spectrum and naturally exhibits a contrasting behavior from the fundamental. [Here we ignore the very small contributions from the higher moment, i.e., $\left\langle Q^{2}(t)\right\rangle$ "squeezed" modulations, of the pump-induced vibrational wave packet. $\left.{ }^{58}\right]$
As seen in the upper left panel of Figure 8, the overtones are peaked at the resonant absorption maximum, where the wave packet passes twice per period of oscillation, and exhibit an amplitude dip at two points near the shoulders of the absorption spectrum. Within this simple homogeneous harmonic model, ${ }^{49}$ the fundamental and overtone phase profiles exhibit a $\pi$ phase change associated with every dip in amplitude, generating the different phase behavior displayed in the upper right panel of Figure 8 . This behavior is clearly not observed for $v_{40}$ and $v_{80}$ in the experimental results reported here (see Figures 5 and 6), where the two modes have nearly equivalent intensities and display similar phase and amplitude profiles.

C. Anharmonic Models. On the other hand, if we consider an asymmetric anharmonic potential for the low-frequency mode, we can explain many of the features observed in the experimental data. The assumption that the motion associated with $v_{40}$ takes place in an asymmetric anharmonic potential is quite reasonable, considering that the iron atom is coordinated only to the histidine on the proximal side, following the pumpinduced ligand photodissociation reaction. As an example, we take the rescaled Morse potential plotted in the inset in the lower right panel of Figure 5. The potential is given by

$$
\begin{gathered}
U_{\mathrm{g}}(q)=D_{\mathrm{e}}\left[1-\mathrm{e}^{-a q}\right]^{2} \\
U_{\mathrm{g}}(q)=\left(\mathrm{e}^{-2 q}-2 \mathrm{e}^{-q}+1\right)
\end{gathered}
$$

with a restoring force:

$$
F(q)=-\frac{\partial U_{\mathrm{g}}}{\partial q}=2\left(\mathrm{e}^{-2 q}-\mathrm{e}^{-q}\right)
$$

where $q=Q-Q_{0}$ is the displacement from equilibrium and $D_{\mathrm{e}}$ and $a$, which scale $U_{\mathrm{g}}$ and $q$, are taken to be unity. The classical equation of motion for $q$ is given by

$$
m \ddot{q}=F(q)-\gamma_{\text {int }} \dot{q}
$$

where $\dot{q}$ is the velocity and $-\gamma_{\text {int }} \dot{q}$ is the velocity dependent damping term. In Figure 9, we give a working example of the anharmonic potential. The factor $-\gamma_{\text {int }} \dot{q}$ in the expression for coordinate motion can be taken to represent the intrinsic homogeneous damping (due to population decay).

To simulate the first moment dynamics of the position coordinate associated with vibrational oscillations in an anharmonic potential, we place a particle in the Morse potential with a large initial displacement (which justifies the classical description). We then follow the dynamics induced by the restoring force $F(q)$ and the intrinsic damping. The simulated oscillatory signal for the homogeneous anharmonic model and the corresponding LPSVD power spectrum are presented in the upper panel of Figure 9. In this case, the only source of signal decay is the inherent damping present in the expression for coordinate motion $\left(-\gamma_{\text {int }} \dot{q}\right)$.

For small initial displacements, the Morse potential can be well approximated as a harmonic potential and the dynamics resembles a simple damped harmonic oscillator. However, higher harmonics are needed to fit the simulated dynamics for large displacements. The relative amplitude of these higher harmonics (compared to the fundamental) increases as the initial displacement gets larger and the particle samples more anharmonic regions of the Morse potential. The intrinsic damping tends to decrease the amplitude of the oscillations so that the particle returns to regions of the potential closer to the equilibrium position, associated with higher frequencies. As a 


\section{Homogeneous Anharmonic Model}

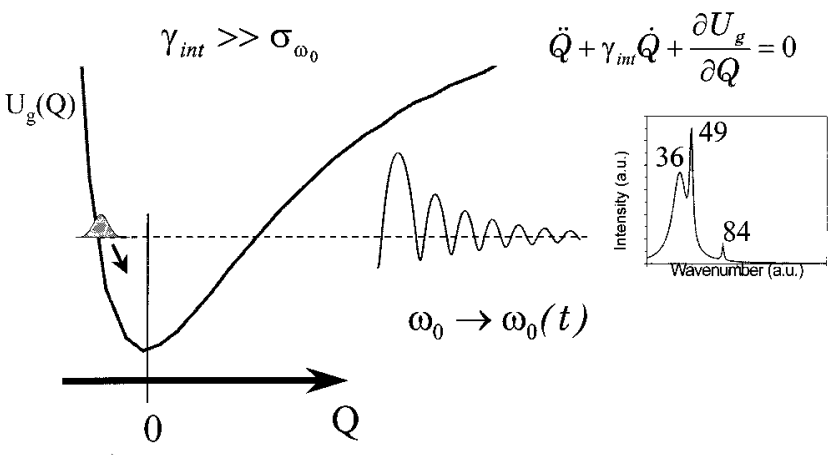

Heterogeneous Anharmonic Model

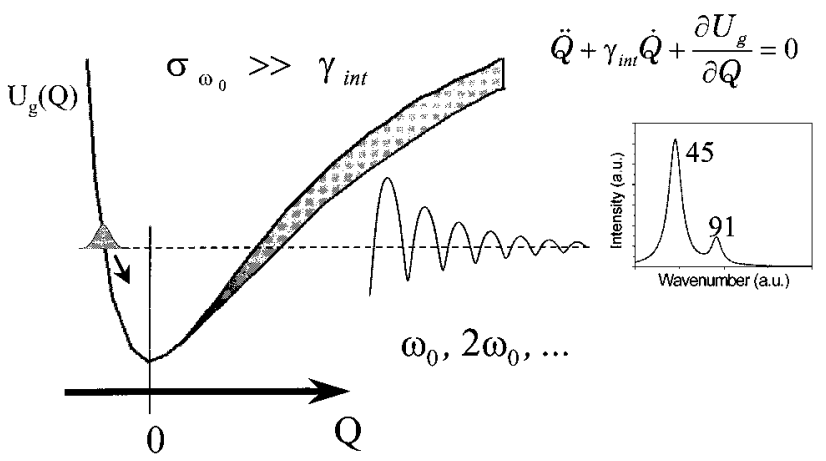

Figure 9. Signals simulated using the dynamics of a particle initially displaced on a Morse potential $\left[U_{\mathrm{g}}(q)=\mathrm{e}^{-2 q}-2 \mathrm{e}^{-q}\right]$, as well as the corresponding power spectra, are presented considering two sources of damping. In the upper panel we consider the inherent homogeneous population damping $\left(\gamma_{\text {int }} \gg \sigma_{\omega_{0}}\right)$ which is analogous to "friction" on the potential surface. Due to the decreasing oscillatory amplitude in the anharmonic potential, the simulated signal contains time dependent frequencies, which are fit with LPSVD using two damped cosine functions with different lifetimes. In the lower panel, the damping factor is introduced as an inhomogeneous frequency distribution $\left(\sigma_{\omega_{0}} \gg \gamma_{\text {int }}\right)$, which is associated with pure dephasing and/or different potential curvatures. The signal simulated using the heterogeneous anharmonic model is fit by LPSVD with a damped fundamental oscillation and its higher harmonics.

result, for large initial displacements, the oscillatory frequency effectively becomes time dependent $\left[\omega_{0} \rightarrow \omega_{0}(t)\right]$, starting at lower frequencies and evolving toward higher frequencies as the system damps (see simulated signal in the upper panel of Figure 9). The LPSVD algorithm fits such a signal with a low frequency and short lifetime component (e.g., $36 \mathrm{~cm}^{-1}$ ) for the early time signal and with a longer-lived higher frequency component (e.g., $49 \mathrm{~cm}^{-1}$ ) for the signal at later times. This generates the power spectrum presented in the inset of the upper panel of Figure 9. Since the experimental signals do not display a time-dependent oscillatory frequency (i.e., a single peak in the power spectrum is sufficient to fit the fundamental oscillation), we are led to an anharmonic model where damping due to the inhomogeneous nature of the system is explicitly considered.

The experimentally observed oscillatory signals can be damped by sources other than population decay. For example, the protein conformational substates discussed earlier lead to correlated distributions of heme out-of-plane geometry and doming frequency. The distribution of vibrational frequencies will broaden the spectral line as well as damp the oscillatory signals. We also note that, since the oscillatory frequency in an anharmonic potential depends on the initial displacement, conformational inhomogeneities can induce a distribution of frequencies through either a distribution of initial displacements or through a distribution in the shape (curvature) of the potential (or both). Finally, if the protein and/or solvent undergo structural fluctuations on faster time scales, pure dephasing ${ }^{59}$ (bath induced energy level fluctuations) will result, which is another form of line broadening without population decay.

As an example, a distribution in the curvature of the potential is presented in the lower panel of Figure 9. In this heterogeneous anharmonic model, the inhomogeneous broadening (or pure dephasing) is considered to be larger than the intrinsic damping due to population decay $\left(\sigma_{\omega_{0}} \gg \gamma_{\text {int }}\right)$. To generate the simulated signal within this model, we allow the system to evolve on the anaharmonic potential with small inherent damping $\left(-\gamma_{\text {int }} \dot{q}\right)$. For the dynamics generated under these conditions, the amplitude of oscillations and, hence, the fundamental oscillatory frequency remain almost constant in time. This means that the coordinate $Q(t)$ can be well described using a Fourier series involving only the fundamental frequency and its higher harmonics $\left(\omega_{0}, 2 \omega_{0}, \ldots\right)$. The final simulated signal is generated by convolving the dynamics of a single protein molecule over a frequency distribution that is commensurate with the experimentally observed peak width in the power spectrum $\left(\sim 12 \mathrm{~cm}^{-1}\right)$.

Within this model, we write the signal for a single undamped anharmonic oscillator as a Fourier series:

$$
S_{\mathrm{h}}\left(\omega_{0}, \omega_{c}, \tau\right)=\sum_{n=1}^{\infty} A_{n} \cos \left(n \omega_{0} \tau+\phi_{n}\right)
$$

Here, the summation is over all the harmonics of the fundamental mode frequency $\omega_{0}$, and the optical carrier frequency dependence of the final signal is denoted by $\omega_{\mathrm{c}}$. For simplicity, we assume that, for a narrow frequency distribution, the amplitudes $A_{n}$ of the various overtones do not depend on the mode frequency. If the inhomogeneous vibrational distribution is specified by a mean vibration frequency $\bar{\omega}_{0}$ and a function $f\left(\bar{\omega}_{0}-\omega_{0}\right)$, the final signal is obtained from a simple convolution of the above expression with the inhomogeneous distribution:

$$
S_{\mathrm{I}}\left(\bar{\omega}_{0}, \omega_{\mathrm{c}}, \tau\right)=\int d \omega_{0} f\left(\bar{\omega}_{0}-\omega_{0}\right) S_{\mathrm{h}}\left(\omega_{0}, \omega_{\mathrm{c}}, \tau\right)
$$

The observed signal is well described by this model, using a fundamental oscillation and its higher harmonic, as seen in the associated LPSVD power spectrum in the lower panels of Figures 5 and 9. However, the damping associated with inhomogeneous broadening can have somewhat stronger effects on the decay times of the higher harmonics. In contrast, pure dephasing affects the damping of the fundamental and its harmonics in a similar fashion. Since the lifetime observed for $v_{80}$ in ferrous cytochrome $c, \mathrm{MbNO}$, and $\mathrm{HbNO}$ is comparable to the lifetime of $v_{40}$, we infer that the damping of the lowfrequency signals probably involves some degree of pure dephasing, along with enough inhomogeneous broadening to account for the frequency drift of $v_{40}$ shown in Figure 4.

If we consider an anharmonic potential for the vibrational coordinate, the first moment modulation $\langle Q(t)\rangle$ in eq 1 directly contains the higher harmonics of the fundamental frequency. Consequently, the centroid of the line shape is modulated at the higher harmonics, as well as at the fundamental frequency. The amplitude and phase profiles of such harmonics, when detected in a pump/probe experiment, are expected to track the amplitude and phase profile of the fundamental, i.e., the 
amplitudes dip and their phase flips by $\pi$ at the absorption maximum. This is illustrated in the lower panels of Figure 8.

The presence of a $\pi$ phase flip and amplitude dip in ferrous cytochrome $c$ around $423 \mathrm{~nm}$ for both $v_{40}$ and $\nu_{80}$ (see Figure 5 ) agrees well with the predictions made using the above model. We have used a Gaussian frequency distribution equivalent to a $\sim 12 \mathrm{~cm}^{-1}$ inhomogeneous broadening of the $45 \mathrm{~cm}^{-1}$ mode to generate the simulated signal presented in the lower panel of Figure 5. The simulations look very similar to the oscillatory signal measured in ferrous cytochrome $c$ at 426 and $434 \mathrm{~nm}$ presented in the third and fourth panels (and to the signal measured at 415 and $420 \mathrm{~nm}$ when the $\pi$ phase shift is added). The similarity is connected to the fixed relative phase relation between $v_{40}$ and $v_{80}$ in the experimental and simulated data as seen in Figure 5. The alignment of the maxima and minima of these oscillatory components is the key condition needed to generate the pattern observed in the experimental data. The good agreement, observed between the experimental data for ferrous cytochrome $c$ and the simulations, makes a strong case for a description of the nuclear coordinate motion using an anharmonic potential surface.

Similar behavior is observed for $v_{40}$ and $v_{80}$ in $\mathrm{MbNO}$, as displayed in Figure 6. The $80 \mathrm{~cm}^{-1}$ mode displays a phase flip and a dip in amplitude near $427 \mathrm{~nm}$. The phase of the $40 \mathrm{~cm}^{-1}$ mode presents a slower $\pi$ phase shift in the $427 \mathrm{~nm}$ region, which is not associated with a clear dip in amplitude. This observation is anomalous and may signal the presence of more complex electron-nuclear coupling (e.g., quadratic coupling), which can shift the phase and amplitude transition points away from the absorption maximum. ${ }^{49}$ The phase drift observed in the 445-480 nm region for both the $v_{40}$ and the $v_{80}$ modes in MbNO (Figure 6) is probably correlated to the frequency drift displayed in the same spectral region. The carrier wavelength dependence of both the frequency and phase may be related to the underlying inhomogeneity associated with the deoxy $\mathrm{Mb}$ Soret band. Thus, the carrier wavelength may resonantly select a subpopulation of conformational states that have different values of $v_{40}$. Such a scenario is consistent with the concept of a distribution of anharmonic potential surfaces that is correlated with the distribution of electronic transition energies.

D. Alternative Models. An alternative possibility is a model recently proposed ${ }^{60}$ to account for the anomalous phase and amplitude behavior of the $47 \mathrm{~cm}^{-1}$ mode, observed in studies of the ultrafast photodissociation of $\mathrm{CO}$ from cytochrome oxidase. This proposal suggests that sequential population transfer from the $\mathrm{CO}$ bound to the $\mathrm{CO}$ unbound state takes place as the heme oscillates (domes) with a period of 700 fs. The unusual phase and amplitude excitation profiles observed for the $47 \mathrm{~cm}^{-1}$ mode of cytochrome oxidase resemble the observations presented here for $v_{40}$ in $\mathrm{Mb}$ and are used by Liebl et al. ${ }^{60}$ to rationalize the coherent population transfer model. However, we also see clear evidence for $v_{40}$ in deoxy $\mathrm{Mb}$, where no reaction or population transfer event is taking place (see Figure 2). Thus, it appears that $v_{40}$ represents a genuine vibrational coherence, and the coherent population transfer model ${ }^{60}$ can be eliminated from consideration (at least for $\mathrm{Mb}$ ). The observation of $v_{40}$ in more sophisticated wavelengthselective modulation experiments ${ }^{57}$ on $\mathrm{MbNO}$, which experimentally discriminate against population transfer signals, is also inconsistent with a coherent population transfer model for NO photodissociation.

The basic assignment (see below) of $v_{40}$ and $v_{80}$ to heme doming $^{6,12,32}$ is in accord with the conclusions of Liebl et al. ${ }^{60}$ However, we differ on the physical mechanism that generates the oscillatory signal. We suggest that the signal is a genuine vibrational coherence, triggered by the electron rearrangements associated with ligand photodissociation, and that a heterogeneous, anharmonic model, analogous to the one presented in the lower portion of Figure 9, is the key to a quantitative understanding of the observations.

E. Anharmonic Mode Assignments. Through the study of a wide variety of heme proteins and model compounds, we have shown that $v_{40}$ involves the core of the porphyrin macrocycle and is affected by the replacement of the heme iron atom with cobalt. This strongly suggests that $v_{40}$ is connected to the heme doming motion. The possibility of a purely electronic coherence, associated with the low-lying spin states of the ferrous heme iron atom cannot be completely excluded at this time. However, such an assignment is difficult to reconcile with the presence of $v_{40}$ in both the ferric and ferrous species and with the appearance of the $v_{80}$ harmonic only in the photolytically excited samples. These observations, along with recent normal mode calculations, ${ }^{29-31}$ which predict the doming frequency near 50 $\mathrm{cm}^{-1}$, strongly support a doming assignment for $v_{40}$ and $v_{80}$. Moreover, the force constant previously estimated ${ }^{25-27}$ from ligand rebinding kinetics $(K \approx 15 \mathrm{~N} / \mathrm{m})$ also predicts a doming frequency on the order of $50 \mathrm{~cm}^{-1}$. The large initial displacements needed within an anharmonic potential model to generate the observed higher harmonics, along with the presence of harmonics such as $v_{80}$ in the photochemically active samples, suggest that $v_{40}$ is a key fundamental oscillation that is directly coupled to the reaction coordinate. The iron motion associated with heme doming, from the in-plane position in the ligand bound state of $\mathrm{Mb}$ to the out-of-plane displaced position $(\sim 0.4$ $\AA$ ) for the unligated state, ${ }^{25-30}$ provides the large initial displacements needed for the appearance of the higher harmonics in a Fourier series description of the anharmonic coordinate motion. As a result of the above considerations, we propose that $v_{40}$ and $v_{80}$ are a direct reflection of the anharmonic potential that describes the heme doming motion and that the damping of the oscillatory signals arises from inhomogeneity and/or pure dephasing effects.

The nearly equal spacing of the harmonic progression $\left(v_{80} \approx\right.$ $2 v_{40}$ ) may appear surprising for an anharmonic potential surface. However, for large displacements from equilibrium, the energy level spacing in an anharmonic Morse potential becomes nearly linear in quantum number for the lower harmonics. This is consistent with the classical wave packet description of the anharmonic motion [i.e., the Fourier series describing $\langle Q(t)\rangle$ will always contain integer multiples of the fundamental frequency]. For the Morse oscillator, the expressions are analytic, since the energy eigenvalues are given by

$$
E_{n}=\left(n+\left(\frac{1}{2}\right)\right) \hbar \omega-\left(n+\left(\frac{1}{2}\right)\right)^{2} \hbar \omega x_{\mathrm{e}}
$$

with

$$
x_{\mathrm{e}}=\frac{a^{2} \hbar}{2 m \omega} \quad a=\sqrt{\frac{m}{2 D_{\mathrm{e}}}} \omega
$$

so that

$E_{n+s}-E_{n}=s \hbar \omega\left[1-(2 n+1) x_{\mathrm{e}}-s x_{\mathrm{e}}\right] \approx s \hbar \omega\left[1-2 n x_{e}\right]$

which is linear in $s$ for $n \gg s$. The linear relation implies that the lower harmonics (smalls) at large displacement (large $n$ ) will be integral multiples of the "fundamental" frequency $\omega\left[1-2 n x_{\mathrm{e}}\right]$. 

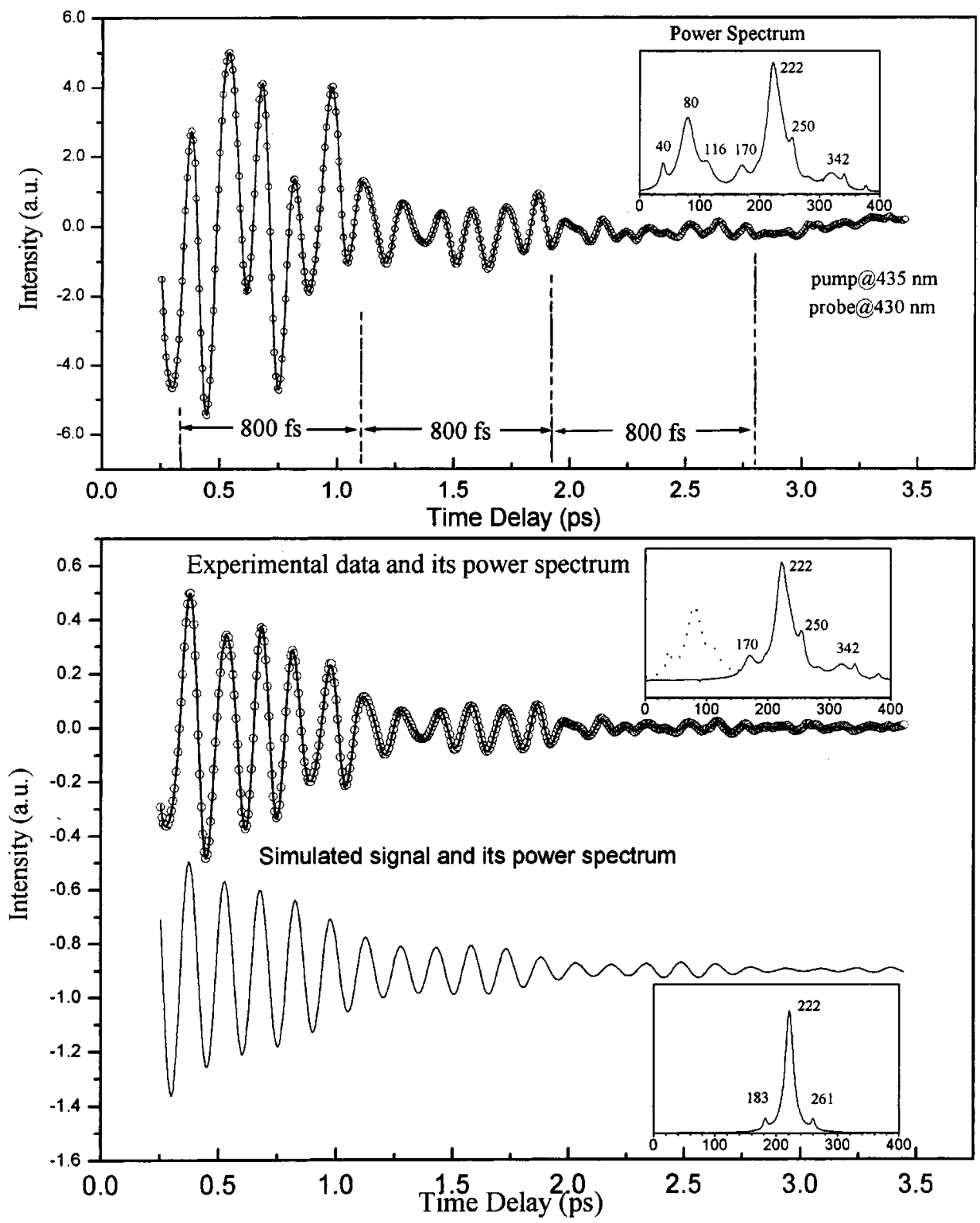

Figure 10. Upper panel displays the oscillatory signal from MbNO-generated using the detuned detection scheme (pump/probe carrier wavelength at $435 \mathrm{~nm}$, detection at $430 \mathrm{~nm}$, for $\sim 60 \mathrm{fs}$ pulses). The vertical dashed lines, defining $800 \mathrm{fs}$ intervals, help one follow the sequential damping of the $220 \mathrm{~cm}^{-1}$ mode. The inset shows the corresponding power spectrum. The lower panel displays the same experimental data as the upper panel, after the lower frequency modes (shown by the dashed line in the associated power spectrum) have been subtracted. A simulated signal in which the damping of a $220 \mathrm{~cm}^{-1}$ oscillatory signal is modulated by a $40 \mathrm{~cm}^{-1}$ oscillation is also shown. The expression used for the simulation is $S_{\mathrm{SIM}}=\cos \left(\omega_{220} t\right) \cdot \exp (-\gamma t) \cdot \exp \left(-\Delta \gamma t\left(1-\cos \left(\omega_{40} t\right)\right)\right)$ where $\gamma=(775 \mathrm{fs})^{-1}$ and $\Delta \gamma=(5.2 \mathrm{ps})^{-1}$. The last inset shows the power spectrum associated with the simulated signal. The sequential damping of the $220 \mathrm{~cm}^{-1}$ oscillations adds two sidebands (at 183 and $261 \mathrm{~cm}^{-1}$ ) to the power spectrum with an appropriate relative phase.

F. Mode Coupling and Damping Effects. The assignment of $v_{40}$ and $v_{80}$ to the heme doming motion is also in agreement with the data presented in Figure 10, which is suggestive of coupling between $v_{\mathrm{Fe}-\mathrm{His}}$, at $220 \mathrm{~cm}^{-1}$, and the $v_{40}$ mode. The upper panel of Figure 10 displays the oscillatory signal generated using detuned detection ${ }^{32}$ to enhance the high-frequency portion of the reaction driven coherence in $\mathrm{MbNO}$. The corresponding power spectrum is presented in the inset. The dominating feature in the data is the $220 \mathrm{~cm}^{-1}$ oscillation associated with the $\mathrm{Fe}-$ His mode. An interesting observation is the sequential damping of the $220 \mathrm{~cm}^{-1}$ oscillations. The oscillations have almost the same amplitude for the initial $\sim 800 \mathrm{fs}$, after which the amplitude drops and remains almost constant for the next $\sim 800 \mathrm{fs}$, and so on, until they damp out. Since the period of oscillation of the $40 \mathrm{~cm}^{-1}$ mode is $800 \mathrm{fs}$, we infer that the damping of the
$220 \mathrm{~cm}^{-1}$ oscillations might be modulated by the motion associated with the $40 \mathrm{~cm}^{-1}$ mode. In the lower panel of Figure 10 , we display the same experimental data as in the upper panel, after subtracting the modes below $150 \mathrm{~cm}^{-1}$ (plotted with dashed line in the second inset). Below, we display a simulated signal that resembles the experimental data, along with its LPSVD generated power spectrum. The expression used to generate the simulated signal contains a $220 \mathrm{~cm}^{-1}$ mode whose damping is modulated by the $40 \mathrm{~cm}^{-1}$ mode:

$$
S_{\mathrm{SIM}}(t)=\mathrm{e}^{-t\left[\gamma_{0}+\Delta \gamma\left(1-\cos \omega_{40} t\right)\right]} \cos \omega_{220} t
$$

where $\omega_{220}$ and $\omega_{40}$ are the angular frequencies of the 220 and $40 \mathrm{~cm}^{-1}$ modes. The damping factor consists of the regular 
exponential decay term, $\mathrm{e}^{-\gamma_{0} t}$, to which we add a term representing the effect of the $40 \mathrm{~cm}^{-1}$ mode on the overall damping:

$$
\exp \left[-\Delta \gamma t\left(1-\cos \omega_{40} t\right)\right]
$$

such that the time-dependent damping factor becomes

$$
\gamma(t)=\gamma_{0}+\Delta \gamma\left(1-\cos \omega_{40} t\right)
$$

The good agreement between the experimental data and the simulations generated by such a simple theoretical expression suggests that there is coupling between the $\mathrm{Fe}-\mathrm{His}$ and the $v_{40}$ doming modes. The presence of such coupling is reasonable since both modes involve the iron motion. For example, when the iron atom displacement passes through the maximum outof-plane position, the $\mathrm{Fe}$-His mode might couple more efficiently with in-plane heme modes (leading to stronger damping of $\left.v_{\mathrm{Fe}-\mathrm{His}}\right)$. When the iron atom is undisplaced and the $\mathrm{Fe}-\mathrm{His}$ mode involves motion that is strictly orthogonal to the heme plane there would be less coupling to in-plane modes and subsequently less damping.

An alternative explanation of the pattern observed in Figure 10 could involve the beating between the $\mathrm{Fe}$-His mode (relative amplitude given by A) and two sideband modes (located at $\sim \pm 40 \mathrm{~cm}^{-1}$ ). However, the phases of all three modes would have to be (coincidently) locked into a specifically required relationship in order to generate the observed oscillatory pattern. Using the expression

$$
\begin{gathered}
\cos \left(\omega_{260} t+\varphi_{220}+\varphi_{40}\right)+\cos \left(\omega_{180} t+\varphi_{220}-\varphi_{40}\right)+ \\
2 A \cos \left(\omega_{220} t+\varphi_{220}\right)= \\
2 \cos \left(\omega_{220} t+\varphi_{220}\right)\left[A+\cos \left(\omega_{40} t+\varphi_{40}\right)\right]
\end{gathered}
$$

we derive the relationship between the phases $\left(\varphi_{260}=\varphi_{220}+\varphi_{40}\right.$ and $\left.\varphi_{180}=\varphi_{220}-\varphi_{40}\right)$ of the two sidebands and the $220 \mathrm{~cm}^{-1}$ mode to be $\varphi_{260}+\varphi_{180}=2 \varphi_{220}$. Since we consider such a coincidence to be highly unlikely, the coupling between the doming and $\mathrm{Fe}-\mathrm{His}$ modes remains as an intriguing source of vibrational damping in this system.

In contrast to the $v_{\mathrm{Fe}-\mathrm{His}}$ mode, which may damp by coupling to the in-plane heme vibrations, the damping of the $40 \mathrm{~cm}^{-1}$ doming motion is probably related to coupling between the heme and the protein. It has been well documented ${ }^{19,21,23,28,48,61-67}$ that $\mathrm{Mb}$ undergoes structural rearrangements following the ligand photodissociation reaction. These rearrangements surely affect the characteristics of the heme active site, including the shape of the potential that governs the heme doming motion. One possibility is that, following the photolysis reaction, the iron atom initially feels a more anharmonic potential that evolves, due to the protein relaxation dynamics, toward a more harmonic potential, associated with the equilibrium structure of deoxy Mb. In Figure 11, we display two example potential surfaces for the iron doming motion. The first one arises immediately after the photolysis reaction (solid line) and, at 300 $\mathrm{K}$, evolves on a picosecond $\left(\tau_{\mathrm{R}}\right)$ time scale toward the more harmonic surface (dashed line) associated with the relaxed deoxy Mb state. For example, the steeper slope at small coordinate values in the early time potential surface could be due to steric hindrance between the proximal histidine and the porphyrin ring. The presence of $v_{80}$ in the reactive samples can be explained by the large initial displacements (and the anharmonic potential) associated with the dissociation reaction. The much smaller displacements and the more harmonic shape of the potential surface in the photostable samples explains the absence of $v_{80}$ and accounts for the similar value of $v_{40}$ in the photostable and

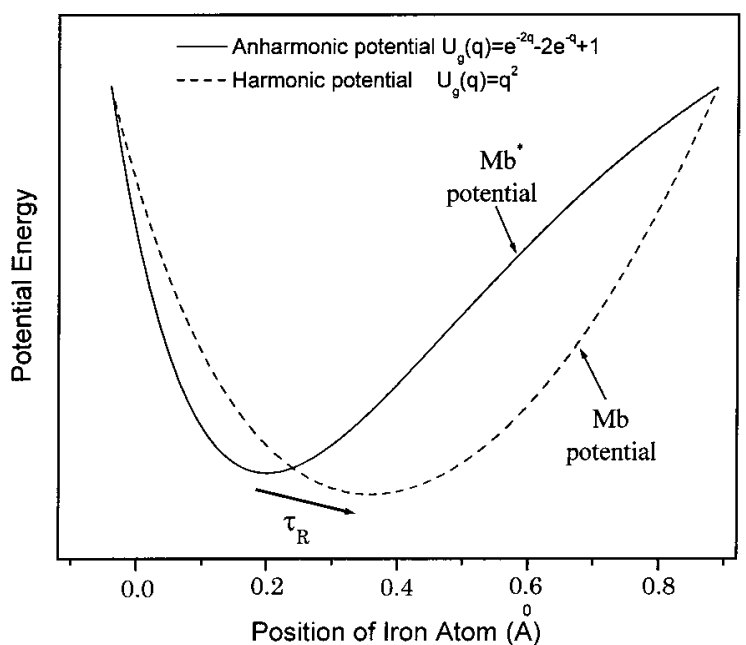

Figure 11. A time dependent potential surface can be associated with the forces acting on the iron atom following the ligand photodissociation. The surfaces for two pump/probe delay times, are displayed. The anharmonic potential (solid line) dictates the $v_{40}$ and $v_{80}$ dynamics immediately after the photodissociation reaction, before any protein structural rearrangements can take place. The more harmonic potential (dashed line) characterizes the $v_{40}$ dynamics for the relaxed structure of deoxy Mb. The evolution of the potential shape induced by the protein structural rearrangements is expected to take place on the picosecond time scale $\left(\tau_{\mathrm{R}}\right)$.

photoactive samples. The early time potential can be associated with the state sometimes denoted as $\mathrm{Mb}^{*}$, which involves a deligated and partially relaxed heme surrounded by a protein that has not yet relaxed from the bound state configuration to its new set of equilibrium states. The relaxation of such states can, in principle, be observed using a combination of short time and low-temperature measurements, and we are currently exploring FCS studies in the low-temperature regime to probe the sensitivity of this technique to the protein/heme relaxation processes.

We note in closing that a mode analogous to $v_{40}$ has not yet been clearly observed ${ }^{68}$ in recent high-resolution synchrotron based experiments on $\mathrm{Mb}$, which are sensitive to the vibrational spectrum of the ${ }^{57} \mathrm{Fe}$ at the active site. A suppression of the intensity of this mode in the detected Fe density-of-states would be explained if the iron motion represents a relatively small fraction of the total kinetic energy involved in the $v_{40}$ motion. This would be the case if there were significant collective motions that involved the surrounding protein or solvent material when the heme domes. ${ }^{68} \mathrm{~A}$ final possibility is that the motion associated with $v_{40}$ is more strongly damped below the glass transition, at the low temperatures used in the synchrotron experiments.

Acknowledgment. This work was supported by grants from the National Institutes of Health DK35090 and the National Science Foundation MCB9904516.

\section{References and Notes}

(1) Scherer, N. F.; Jonas, D. M.; Fleming, G. R. J. Chem. Phys. 1993, 99, 153-168.

(2) Pugliano, N.; Szarka, A. Z.; Hochstrasser, R. M. J. Chem. Phys. 1996, 104, 5062-5079.

(3) Chachisvilis, M.; Fidder, H.; Sundstrom, V. Chem. Phys. Lett. 1995, $234,141-150$.

(4) Polanyi, J. C.; Zewail, A. H. Acc. Chem. Res. 1995, 28, 119-132.

(5) Ziegler, L. D.; Fan, R.; Desrosiers, A. E.; Scherer, N. F. J. Chem. Phys. 1994, 100, 1823-1839. 632. 
(7) Vos, M. H.; Rappaport, F.; Lambry, J. C.; Breton, J.; Martin, J. L. Nature 1993, 363, 320-325.

(8) Ruhman, S.; Joly, A. G.; Nelson, K. A. J. Chem. Phys. 1987, 86, $6563-6565$

(9) Zewail, A. H. Femtochemistry: Ultrafast Dynamics of the Chemical Bond, World Scientific Publishing Co. Pte. Ltd.: Singapore, 1994.

(10) Wang, Q.; Schoenlein, R. W.; Peteanu, L. A.; Mathies, R. A.; Shank,

C. V. Science 1994, 266, 422-424.

(11) Zhu, L. Y.; Wang, W.; Sage, J. T.; Champion, P. M. J. Raman Spectrosc. 1995, 26, 527-534.

(12) Champion, P. M.; Rosca, F.; Wang, W.; Kumar, A. T. N.; Christian, J.; Demidov, A. Laser Techniques for Condensed-Phase and Biological Systems. Proc. SPIE 1998, 3273, 80-89.

(13) Janes, S. M.; Dalickas, G. A.; Eaton, W. A.; Hochstrasser, R. M. Biophys. J. 1988, 54, 545-549.

(14) Ansari, A.; Jones, C. M.; Henry, E. R.; Hofrichter, J.; Eaton, W. A. Biophys. J. 1993, 64, 852-868.

(15) Jackson, T. A.; Lim, M.; Anfinrud, P. A. Chem. Phys. 1994, 180 , $131-140$.

(16) Kaminaka, S.; Ogura, T.; Kitagawa, T. J. Am. Chem. Soc. 1990, $112,23-27$

(17) Lambright, D. G.; Balasubramanian, S.; Boxer, S. G. Chem. Phys. 1991, 158, 249-260.

(18) Nakabayashi, T.; Okamoto, H.; Tasumi, M. J. Phys. Chem. A 1997, $101,3494-3500$

(19) Tian, W. D.; Sage, J. T.; Srajer, V.; Champion, P. M. Phys. Rev. Lett. 1992, 68, 408-411.

(20) Srajer, V.; Reinisch, L.; Champion, P. M. Biochemistry 1991, 30, $4886-4895$

(21) Sage, J. T.; Schomacker, K. T.; Champion, P. M. J. Phys. Chem. 1995, 99, 3394-3405.

(22) Walda, K. N.; Liu, X. Y.; Sharma, V. S.; Magde, D. Biochemistry 1994, 33, 2198-2209.

(23) Austin, R. H.; Beeson, K.; Eisenstein, L.; Frauenfelder, H.; Gunsalus, I. C. Biochemistry 1975, 14, 5355-5373.

(24) Carver, T. E.; Rohlfs, R. J.; Olson, J. S.; Gibson, Q. H.; Blackmore, R. S.; Springer, B. A.; Sligar, S. G. J. Biol. Chem. 1990, 265, 2000720020

(25) Srajer, V.; Reinisch, L.; Champion, P. M. J. Am. Chem. Soc. 1988 , $110,6656-6670$.

(26) Srajer, V.; Champion, P. M. Biochemistry 1991, 30, 7390-7402.

(27) Champion, P. M. J. Raman Spectrosc. 1992, 23, 557-567.

(28) Perutz, M. F. Proc. R. Soc. London B 1980, 208, 135-162

(29) Kozlowski, P. M.; Spiro, T. G.; Berces, A.; Zgierski, M. Z. J. Phys.

Chem. B 1998, 102, 2603-2608.

(30) Spiro, T. G.; Kozlowski, P. M.; Zgierski, M. Z. J. Raman Spectrosc. 1998, 29, 869-879.

(31) Kozlowski, P. M.; Spiro, T. G.; Zgierski, M. Z. J. Phys. Chem. B 2000, 104, 10659-10666.

(32) Rosca, F.; Kumar, A. T. N.; Ye, X.; Sjodin, T.; Demidov, A. A.; Champion, P. M. J. Phys. Chem. A 2000, 104, 4280-4290.

(33) Wang, W.; Demidov, A. A.; Ye, X.; Christian, J. F.; Sjodin, T.; Champion, P. M. J. Raman Spectrosc. 2000, 31, 99-105.

(34) Gerber, N. C.; Sligar, S. G. J. Biol. Chem. 1994, 269, 4260-4266.

(35) Barrick, D.; Dahlquist, F. W. Proteins: Struct., Funct., Genet. 2000 39, 278-290.

(36) Barrick, D. Proteins: Struct., Funct., Genet. 2000, 39, 291-308.

(37) Yonetani, T.; Yamamoto, H.; Woodrow, G. V. J. Biol. Chem. 1974, $249,682-690$.
(38) Neya, S.; Funasaki, N.; Sato, T.; Igarashi, N.; Tanaka, N. J. Biol Chem 1993, 268, 8935-8942.

(39) Mazumdar, S. J. Chem. Soc., Dalton Trans. 1991, 2091.

(40) Wise, F.; Rosker, M.; Millhauser, G.; Tang, C. L. J. Quantum Electron. 1987, $O E-23,1116$

(41) Kumar, A. T. N.; Zhu, L.; Christian, J. F.; Demidov, A. A.; Champion, P. M. J. Phys. Chem. B 2001, 105, 7847-7856.

(42) Constantine, S.; Zhou, Y.; Morais, J.; Ziegler, L. D. J. Phys. Chem. A 1997, 101, 5456-5462.

(43) Champion, P. M.; Lange, R. J. Chem. Phys. 1980, 73, 5947-5957.

(44) Adar, F.; Gouterman, M.; Aronowitz, S. J. Phys. Chem. 1976, 80, $2184-2191$

(45) Champion, P. M.; Perreault, G. J. J. Chem. Phys. 1981, 75, 490491.

(46) Friedman, J. M.; Rousseau, D. L. Chem. Phys. Lett. 1978, 55, 488492.

(47) Hochstrasser R. M. Probes of Structure and Function of Macromolecules and Membranes; Academic Press: New York, 1971; p 57.

(48) Demidov, A. A.; Ye, X.; Rosca, F.; Kumar, A. T. N.; Ionascu, D.;

Wang, W.; Zhu, L.; Champion, P. M. Manuscript in preparation.

(49) Kumar, A. T. N.; Rosca, F.; Widom, A.; Champion, P. M. J. Chem

Phys. 2001, 114, 701-724.

(50) Kitagawa T. Biological Applications of Raman Spectroscopy;

Wiley-Interscience Publication: New York, 1988; Chapter 3, pp 97-131.

(51) Srajer, V.; Schomacker, K. T.; Champion, P. M. Phys. Rev. Lett. 1986, $57,1267-1270$.

(52) Bangcharoenpaurpong, O.; Schomacker, K. T.; Champion, P. M. J. Am. Chem. Soc. 1984, 106, 5688-5698.

(53) Srajer, V. Ph.D. Thesis, Northeastern University, 1991.

(54) Wang, W.; Ye, X.; Demidov, A. A.; Rosca, F.; Sjodin, T.; Cao, W.; Sheeran, M.; Champion, P. M. J. Phys. Chem. B 2000, 104, 1078910801.

(55) Desbois, A.; Momenteau, M.; Loock, B.; Lutz, M. Spectroscopy Lett. 1981, 14, 257-269.

(56) Rosca, F.; Ionascu, D.; Kumar, A. T. N.; Demidov, A. A.; Champion, P. M. Chem. Phys. Lett. 2001, 337, 107-116.

(57) Rosca, F.; Kumar, A. T. N.; Ionascu, D.; Sjodin, T.; Demidov, A

A.; Champion, P. M. J. Chem. Phys. 2001, 114, 10884-10898.

(58) Kumar, A. T. N.; Rosca, F.; Widom, A.; Champion, P. M. J. Chem Phys. 2001, 114, 6795-6815.

(59) Tokmakoff, A.; Fayer, M. D. Acc. Chem. Res. 1995, 28, 437-445.

(60) Liebl, U.; Lipowski, G.; Negrerie, M.; Lambry, J. C.; Martin, J.

L.; Vos, M. H. Nature 1999, 401, 181-184.

(61) Deak, J.; Chin, H. L.; Lewis, C. M.; Miller, R. J. D. J. Phys. Chem. $B$ 1998, 102, 6621-6634.

(62) Friedman, J. M.; Scott, T. W.; Fisanick, G. J.; Simon, S. R.; Findsen,

E. W.; Ondrias, M. R.; Macdonald, V. W. Science 1985, 229, 187-190.

(63) Petrich, J. W.; Martin, J. L.; Houde, D.; Poyart, C.; Orszag, A. Biochemistry 1987, 26, 7914-7923.

(64) Lingle, R.; Xu, X. B.; Zhu, H. P.; Yu, S. C.; Hopkins, J. B. J. Phys. Chem. 1991, 95, 9320-9331.

(65) Lim, M.; Jackson, T. A.; Anfinrud, P. A. Proc. Natl. Acad. Sci. U.S.A. 1993, 90,8302.

(66) Causgrove, T. P.; Dyer, R. B. Biochemistry 1993, 32, 11985-11991.

(67) Ansari, A.; Jones, C. M.; Henry, E. R.; Hofrichter, J.; Eaton, W. A. Science 1992, 256, 1796-1798.

(68) Sage, J. T.; Durbin, S. M.; Sturhahn, W.; Wharton, D. C.; Champion, P. M.; Hession, P.; Sutter, J.; Alp, E. E. Phys. Rev. Lett. 2001, $86,4966-4969$. 